Narrative Review

\title{
Analgesic Efficacy and Adverse Effects of Meperidine in Managing Postoperative or Labor Pain: A Narrative Review of Randomized Controlled Trials
}

\author{
Stanley Sau Ching Wong, MBBS, and Chi Wai Cheung, MD
}

From: Laboratory and Clinical Research Institute for Pain, Department of Anaesthesiology, Li Ka Shing Faculty of Medicine, The University of Hong Kong, Hong Kong, China

Address Correspondence: Chi Wai Cheung, MD Room 424, Block K Queen Mary Hospital 102, Pokfulam Road Hong Kong

E-mail: cheucw@hku.hk

Disclaimer: Supported by the Department of Anaesthesiology

at the university where the study was conducted.Conflict of interest: Each author certifies that he or she, or a member of his or her immediate family, has no commercial association (i.e., consultancies, stock ownership, equity interest, patent/licensing arrangements, etc.) that might

pose a conflict of interest in connection with the submitted manuscript.

Manuscript received: 06-05-2019 Revised manuscript received: o8-27-2019

Accepted for publication: 09-06-2019

Free full manuscript: www.painphysicianjournal.com
Background: Meperidine, a synthetic opioid, has a rapid onset and short duration of action. Mounting evidence has challenged meperidine's analgesic benefits, and concerns have been raised about its safety profile. Despite recommendations to restrict the prescription of meperidine, the drug remains frequently used.

Objectives: The aim of this study was to evaluate the evidence regarding the efficacy and safety of meperidine for acute postoperative and labor pain.

Study Design: This was a narrative review of the analgesic efficacy and side effects of meperidine compared to other analgesic drugs for acute postoperative and labor pain in adults.

Setting: Randomized controlled trials that compared the analgesic efficacy and side effect profile of meperidine versus another analgesic drug in adult patients were evaluated.

Methods: A systemized search of randomized controlled trials studying meperidine for acute postoperative or labor pain in the adult patient population from PubMed, Medline, and EMBASE was performed. Included studies reported on different routes of meperidine administration including intramuscular, intravenous, and patient-controlled analgesia in various surgical procedures such as abdominal surgery, Cesarean section, gynecological surgery, orthopedic surgery, cardiothoracic surgery, as well as for labor analgesia. Meperidine's analgesic efficacy and safety profile were compared to other opioids (morphine, tramadol, fentanyl, buprenorphine, nalbuphine, and pentazocine), nonsteroidal anti-inflammatory drugs (ketorolac, diclofenac, and indomethacin), dipyrone, ketamine, and bupivacaine.

Results: A total of 62 randomized controlled trials published between 1972 and 2018 were reviewed. Meperidine had a similar or inferior analgesic efficacy compared to other analgesics for acute postoperative or labor pain. Meperidine was associated with more sedation and respiratory depression.

Limitations: The sample sizes of many clinical studies were small, and therefore probably insufficiently powered to detect differences in uncommon side effects, such as central nervous system toxicity. In addition, some of the included clinical studies were old.

Conclusion: Considering the availability of other effective analgesics with potentially fewer side effects, the use of meperidine for acute postoperative or labor pain should not be recommended.

Key words: Acute postoperative pain, adverse effects, labor analgesia, meperidine, pethidine

Pain Physician 2020: 23:175-201 
P oor acute postoperative pain control is associated with reduced patient satisfaction, delayed recovery, increased morbidity, progression to chronic pain, and overall reduced quality of life (1-4). Opioids are commonly used for the management of acute postoperative pain, and are administered via the oral, sublingual, or parenteral [intramuscular (IM), subcutaneous (SC), intravenous (IV)] routes. Frequently used opioids include fentanyl, meperidine, morphine, hydromorphone, and oxycodone $(5,6)$.

Meperidine was the first synthetic opioid used in humans (7). Oral and injectable formulations are available, and the latter is used subcutaneously, intramuscularly, intravenously, and for neuraxial anesthesia. Typical dosages range from $50 \mathrm{mg}$ to $150 \mathrm{mg}$ orally, $25 \mathrm{mg}$ to $100 \mathrm{mg}$ via SC or IM injection, and $25 \mathrm{mg}$ to $50 \mathrm{mg}$ IV, with repeated doses every 3 to 4 hours as necessary $(8,9)$. Oral meperidine is subject to extensive first-pass metabolism resulting in low oral bioavailability, and this is rarely used (10).

Although early reports attested to the analgesic efficacy and safety of meperidine (11), closer scrutiny revealed these were mostly only case reports (10). Advantages of meperidine include its rapid onset and short duration of action (7). However, there is growing evidence against the alleged benefits of meperidine, and concerns have been raised regarding its safety profile $(4,12,13)$. In addition to typical opioid-related side effects such as constipation and drowsiness, meperidine has also been associated with serious side effects including central nervous system excitation and serotonin syndrome (10). It is also associated with intoxicating effects, making it considerably addictive (10). These concerns have led to guidelines and recommendations advocating the use of other opioid drugs and the removal of meperidine from health-system formularies (7,14-16).

Despite recommendations against meperidine prescription, the drug is still frequently used in many parts of the world $(17,18)$. A study showed that 1 in 8 older surgical patients were prescribed meperidine (17). This suggests that awareness and knowledge about the potential problems and analgesic efficacy of meperidine may still be lacking amongst the overall population of health care professionals. This may be worsened by the fact that there is currently a lack of review of clinical evidence on the efficacy, tolerability, and safety of meperidine for postoperative pain management. Previous reviews of meperidine in acute postoperative pain included only a small number of studies $(10,19)$, or only looked at certain routes of administration (19). Therefore, we performed a narrative review of published randomized controlled trials that evaluated the analgesic efficacy and adverse effects of meperidine for acute postoperative pain and labor pain management.

\section{Methods}

\subsection{Literature Search Strategy}

A literature search in PubMed, Medline, and EMBASE databases was performed using the following keywords: ("meperidine" or "pethidine") and ("operation," "surgery," "postoperative," "operative," or "surgical") in combination with ("pain," "analgesic," or "analgesia") and ("safety," "safe," "toxicity," "adverse effect," or "adverse event") and ("efficacy," "effect," or "effectiveness"). Randomized controlled trials published in peer-reviewed journals were included. The language was restricted to English. Papers investigating meperidine in pediatric or adolescent populations were excluded. Given the search was not restricted by publication date, papers that could no longer be retrieved on the internet or hospital library were excluded. All original papers on human clinical studies published up to the period December 2018 were included.

\subsection{Studies Selection}

The initial literature search yielded 575 publications from PubMed and 845 from EMBASE/Medline (Fig. 1). After removal of duplicates, 1,138 articles were assessed; of these, 128 articles fulfilled the inclusion criteria based on title and abstract. These articles were then independently evaluated against the inclusion criteria by 2 investigators, and disagreements between the investigators were discussed and resolved. A total of 62 studies were included for the final analysis. Considering the heterogeneity in methodology and treatment indications among studies published between 1972 and 2018, a narrative review of these studies was performed.

\subsection{Studies Characteristics}

The selected studies were randomized controlled trials. Pain intensity was measured by visual analog scores (VAS - 10cm/100mm, 150mm), verbal rating scales, pain intensity difference, summed pain intensity difference scores, total relief scores, or as 3-, 4-, or 5 -point pain scales. Some studies used both observerrated and patient-rated assessments of pain. Treatment-associated adverse effects were also extracted 
from these included studies.

Meperidine was given via IM injections, IV injections, a combination of IM and IV injections, IV patient-controlled analgesia (PCA), epidural infusions, patient-controlled epidural analgesia, intraarticular (IA) administration, intraperitoneal injection, SC injections, and SC infusions.

\subsection{Inclusion and Exclusion Criteria}

This review included randomized controlled trials that compared the analgesic efficacy and safety of meperidine to other analgesics for acute postoperative or labor pain in adult patients. Clinical studies that administered meperidine as medication for chronic pain, in the pediatric population, in oral formulation, and in the emergency room other than for surgery were excluded. Animal studies were excluded. Studies that compared different routes of meperidine in the absence of another comparator drug or placebo were ineligible for inclusion in this review. Studies not published in English, and those presented or published at conferences, revisions, editorials, or opinion articles were not included.

The data of included studies were extracted and tabulated to allow qualitative comparisons of interventions. The outcome measures chosen for comparison were analgesic efficacy (pain scores), adverse effects, and patient satisfaction.

\section{Review and Results}

\subsection{Analgesic Efficacy by Surgical Setting}

\subsubsection{Abdominal Surgery}

Fifteen studies compared the postoperative pain relief of meperidine with other analgesics after abdominal surgery (Table 1 ) and found that meperidine was associated with higher VAS pain scores (20-22). In one older study that included both patients who underwent abdominal surgery (specifically lower abdominal gynecological surgery) and those who had Cesarean section, analgesia provided by epidural meperidine was not significantly different from epidural bupivacaine (23). Epidural bupivacaine in combination with fentanyl resulted in significantly lower pain scores compared to IM meperidine after colorectal cancer surgery (24).
Literature search of Pubmed/Embase/Medline

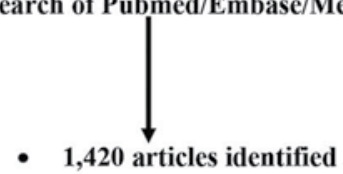

(Pubmed: 575)

(Embase/Medline: 845 )

Exclusion of duplicate citations $(\mathrm{n}=\mathbf{2 8 2})$

1,138 articles reviewed based on title and abstract

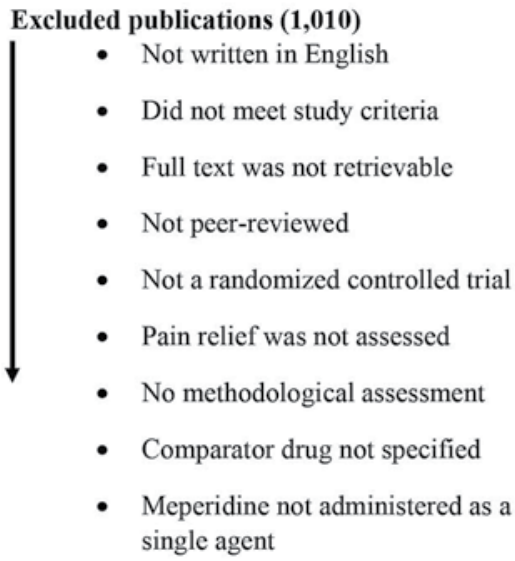

128 full-text articles reviewed

Excluded publications (66)

- Studies comparing between different administration routes of meperidine

- Studies comparing to placebo, not to an active comparator

- Studies that could not be retrieved in full text

- Studies not written in English

- Studies in pediatric patients

- Disagreement between reviewers on including studies for further analysis

62 full-text articles reviewed and included in review

Fig. 1. Flow chart of the literature search and selection of studies

Two studies comparing meperidine to morphine revealed that patients on meperidine experienced significantly more pain $(25,26)$. The first study compared IM meperidine to PCA morphine after vertical gastroplasty, while the second study compared PCA meperidine to PCA morphine for major ab- 
Table 1. Randomized controlled trials of meperidine in managing pain after abdominal surgery

\begin{tabular}{|c|c|c|c|c|c|c|c|}
\hline $\begin{array}{c}\text { Author (yr) } \\
\text { [citation] }\end{array}$ & $\begin{array}{c}\text { Comparative } \\
\text { Drug }\end{array}$ & $\begin{array}{c}\text { Dose, Dosing } \\
\text { Frequency and } \\
\text { Administration } \\
\text { Routes } \\
\end{array}$ & Surgery Type & $\begin{array}{c}\text { Number } \\
\text { of } \\
\text { Patients }\end{array}$ & $\begin{array}{c}\text { Mean } \\
\text { Age } \\
\text { [Gender] }\end{array}$ & Analgesic Results & Adverse Event \\
\hline $\begin{array}{l}\text { Salman et al } \\
\text { (2013) [20] }\end{array}$ & Bupivacaine & $\begin{array}{l}\text { IV PCA meperidine } \\
\text { (loading and } \\
\text { infusion doses at } \\
0.1 \mathrm{~mL} / \mathrm{kg} \text { ) } \\
\text { Epidural } \\
\text { bupivacaine } \\
\text { (loading and } \\
\text { infusion doses at } \\
0.1 \mathrm{~mL} / \mathrm{kg} \text { ) }\end{array}$ & $\begin{array}{l}\text { Abdominal } \\
\text { aortic surgery } \\
\text { (elective) }\end{array}$ & 80 & $\begin{array}{l}59.4 \pm 9.7 \\
{[\mathrm{M} / \mathrm{F}=} \\
38 / 2] \\
61.7 \pm 8.1 \\
{[\mathrm{M} / \mathrm{F}=} \\
39 / 1]\end{array}$ & $\begin{array}{l}\text { Epidural bupivacaine } \\
\text { had lower sedation } \\
\text { and VAS scores than } \\
\text { IV meperidine }(P< \\
.05 \text { each). }\end{array}$ & $\begin{array}{l}\text { Postoperative nausea } \\
\text { was more prevalent in } \\
\text { the meperidine group } \\
(P<.05) \text {. }\end{array}$ \\
\hline $\begin{array}{l}\text { Raddi et al } \\
\text { (2009) [21] }\end{array}$ & Bupivacaine & $\begin{array}{l}\text { IV meperidine (1 } \\
\mathrm{mg} / \mathrm{kg}) \\
\text { Bupivacaine } 0.5 \% \\
\text { injected intra- } \\
\text { pleurally }(20 \mathrm{~mL})\end{array}$ & $\begin{array}{l}\text { Open } \\
\text { cholecystectomy }\end{array}$ & 100 & $\begin{array}{l}45.72 \pm \\
7.12 \\
{[\mathrm{M} / \mathrm{F}=} \\
20 / 30] \\
45.68 \pm \\
7.93 \\
{[\mathrm{M} / \mathrm{F}=} \\
21 / 29]\end{array}$ & $\begin{array}{l}\text { At } 30 \text { min after drug } \\
\text { administration, IV } \\
\text { meperidine had a } \\
\text { significantly higher } \\
\text { mean VAS score } \\
(P<.01) \text {. At every } \\
\text { subsequent time } \\
\text { interval (30-360 } \\
\text { min), IV meperidine } \\
\text { consistently had } \\
\text { significantly higher } \\
\text { mean VAS scores }(P \\
<.001) \text {. }\end{array}$ & $\begin{array}{l}\text { There were no } \\
\text { clinical complications } \\
\text { for bupivacaine. } \\
18 \% \text { of the IV } \\
\text { meperidine group } \\
\text { had postoperative } \\
\text { nausea and vomiting. }\end{array}$ \\
\hline $\begin{array}{l}\text { Ebrahimifard } \\
\text { et al (2013) } \\
\text { [22] }\end{array}$ & Bupivacaine & $\begin{array}{l}\text { IV meperidine (25 } \\
\text { mg) } \\
\text { IP } 0.5 \% \\
\text { bupivacaine ( } 20 \\
\mathrm{~cm} 3)\end{array}$ & $\begin{array}{l}\text { Laparoscopic } \\
\text { cholecystectomy }\end{array}$ & 48 & $\begin{array}{l}47 \pm 11 \\
{[\mathrm{M} / \mathrm{F}=} \\
4 / 16] \\
49 \pm 10 \\
{[\mathrm{M} / \mathrm{F}=} \\
5 / 15]\end{array}$ & $\begin{array}{l}\text { At } 1,4 \text {, and } 8 \text { hrs after } \\
\text { surgery, VAS pain } \\
\text { scores } \\
\text { were significantly } \\
\text { lower with bupivacaine } \\
\text { than with meperidine } \\
(P=.035, .048 \text {, and } \\
.009 \text {, respectively). } \\
\text { There was no } \\
\text { significant difference } \\
\text { between the groups } 24 \\
\text { hrs after the procedure } \\
(P=.223) \text {. }\end{array}$ & $\begin{array}{l}\text { Nausea and vomiting } \\
\text { were lower in the } \\
\text { bupivacaine group. }\end{array}$ \\
\hline $\begin{array}{l}\text { Brownridge et } \\
\text { al (1985) [23] }\end{array}$ & Bupivacaine & $\begin{array}{l}\text { IM meperidine } \\
(100 \mathrm{mg}) \\
\text { Epidural } \\
\text { meperidine (50 } \\
\mathrm{mg}) \\
\text { Epidural } \\
\text { bupivacaine (25 } \\
\mathrm{mg})\end{array}$ & $\begin{array}{l}\text { Lower } \\
\text { abdominal } \\
\text { surgery } \\
\text { (The study } \\
\text { also included } \\
\text { patients with } \\
\text { Cesarean } \\
\text { section.) } \\
\end{array}$ & 19 & $\begin{array}{l}\text { NS } \\
\text { (All } \\
\text { patients } \\
\text { were } \\
\text { female in } \\
\text { this study.) }\end{array}$ & $\begin{array}{l}\text { Analgesia provided by } \\
\text { epidural meperidine } \\
50 \text { mg was superior to } \\
\text { IM meperidine } 100 \\
\mathrm{mg}(P<.05) \text { but not } \\
\text { statistically better than } \\
\text { epidural bupivacaine. }\end{array}$ & $\begin{array}{l}\text { There were no } \\
\text { clinically significant } \\
\text { differences in sedation, } \\
\text { nausea, itching, } \\
\text { respiratory rate, or } \\
\text { blood pressure. }\end{array}$ \\
\hline $\begin{array}{l}\text { Rimaitis et al } \\
(2003)[24]\end{array}$ & $\begin{array}{l}\text { Bupivacaine } \\
\text { with fentanyl }\end{array}$ & $\begin{array}{l}\mathrm{IM} \text { meperidine }(0.5 \\
\mathrm{mg} / \mathrm{kg}) \text { every } 4 \mathrm{hrs} \\
\text { Epidural } \\
\text { continuous } \\
\text { infusion } \\
\text { bupivacaine }(1.0 \\
\mathrm{mg} / \mathrm{mL}) \text { with } \\
\text { fentanyl }(5 \mathrm{mg} / \\
\mathrm{mL}) \text { at a rate of 3-6 } \\
\mathrm{mL} / \mathrm{hr}\end{array}$ & $\begin{array}{l}\text { Colorectal } \\
\text { cancer surgery }\end{array}$ & 100 & $\begin{array}{l}67 \pm 11 \\
{[\mathrm{M} / \mathrm{F}=} \\
24 / 26] \\
65 \pm 13 \\
{[\mathrm{M} / \mathrm{F}=} \\
25 / 25]\end{array}$ & $\begin{array}{l}\text { VAS pain scores at } \\
\text { rest and on coughing } \\
\text { were significantly } \\
\text { better in the epidural } \\
\text { analgesia group than } \\
\text { in the systemic IM } \\
\text { meperidine analgesia } \\
\text { group }(P<.05) \text {. }\end{array}$ & $\begin{array}{l}\text { The meperidine group } \\
\text { had more sedation, } \\
\text { nausea and vomiting } \\
(P<.05) \text {. } \\
\text { The bupivacaine group } \\
\text { had more pruritus ( } P \\
<.05) \text {. } \\
\text { No statistically } \\
\text { significant } \\
\text { differences }(P=.62) \\
\text { in postoperative } \\
\text { complications were } \\
\text { found between the } 2 \\
\text { groups. }\end{array}$ \\
\hline
\end{tabular}


Table 1 (cont.). Randomized controlled trials of meperidine in managing pain after abdominal surgery

\begin{tabular}{|c|c|c|c|c|c|c|c|}
\hline $\begin{array}{c}\text { Author (yr) } \\
\text { [citation] }\end{array}$ & $\begin{array}{c}\text { Comparative } \\
\text { Drug }\end{array}$ & $\begin{array}{c}\text { Dose, Dosing } \\
\text { Frequency and } \\
\text { Administration } \\
\text { Routes }\end{array}$ & Surgery Type & $\begin{array}{c}\text { Number } \\
\text { of } \\
\text { Patients }\end{array}$ & $\begin{array}{c}\text { Mean } \\
\text { Age } \\
\text { [Gender] }\end{array}$ & Analgesic Results & Adverse Event \\
\hline $\begin{array}{l}\text { Kyzer et al } \\
\text { (1995) [25] }\end{array}$ & Morphine & $\begin{array}{l}\text { IM meperidine } \\
\text { ( } 50-100 \mathrm{mg} \text { ) every } \\
3-4 \text { hrs as needed } \\
\text { IV PCA morphine } \\
\text { ( } 5 \mathrm{mg} \text {, lockout } \\
\text { interval } 5 \text { min in } \\
\text { recovery room, } \\
\text { followed by } 2 \\
\text { mg dose lockout } \\
\text { interval } 15 \text { min) }\end{array}$ & $\begin{array}{l}\text { Sialastic } \\
\text { ring vertical } \\
\text { gastroplasty }\end{array}$ & 23 & $\begin{array}{l}37 \\
(19-42)^{*} \\
{[\mathrm{M} / \mathrm{F}=} \\
4 / 7] \\
34 \\
(22-56)^{*} \\
{[\mathrm{M} / \mathrm{F}=} \\
5 / 7]\end{array}$ & $\begin{array}{l}\text { The difference in the } \\
\text { amount of narcotics } \\
\text { used between } \\
\text { the groups was } \\
\text { statistically significant } \\
(P<.005) \text {. } \\
\text { Significantly higher } \\
\text { pain scores were seen } \\
\text { in the meperidine } \\
\text { group }(P<.01) \text {. }\end{array}$ & $\begin{array}{l}\text { There was a } \\
\text { significantly higher } \\
\text { incidence of wound } \\
\text { infection in the IV } \\
\text { PCA morphine } \\
\text { group (no } P \text { value). }\end{array}$ \\
\hline $\begin{array}{l}\text { Plummer et al } \\
\text { (1997) [26] }\end{array}$ & Morphine & $\begin{array}{l}\text { IV PCA } \\
\text { meperidine (bolus } \\
\text { doses of } 9,12 \text {, or } \\
18 \mathrm{mg} \text { ) } \\
\text { IV PCA morphine } \\
\text { (bolus doses of } \\
0.75,1.0 \text {, or } 1.5 \mathrm{mg} \text { ) }\end{array}$ & $\begin{array}{l}\text { Major } \\
\text { abdominal } \\
\text { surgery }\end{array}$ & 102 & $\begin{array}{l}63 \\
(23-86)^{*} \\
{[\mathrm{M} / \mathrm{F}=} \\
25 / 25] \\
62 \\
(24-89)^{*} \\
{[\mathrm{M} / \mathrm{F}=} \\
24 / 28]\end{array}$ & $\begin{array}{l}\text { Pain on sitting }(P= \\
.037) \text { but not pain } \\
\text { at rest }(P=.8) \text { was } \\
\text { significantly less in } \\
\text { patients receiving } \\
\text { morphine. }\end{array}$ & $\begin{array}{l}\text { Severity of nausea, } \\
\text { mood disturbances, } \\
\text { and incidence of } \\
\text { unusual dreams } \\
\text { did not differ } \\
\text { significantly between } \\
\text { the drugs. }\end{array}$ \\
\hline $\begin{array}{l}\text { Woodhouse } \\
\text { et al (1999) } \\
{[27]}\end{array}$ & $\begin{array}{l}\text { Morphine } \\
\text { Fentanyl }\end{array}$ & $\begin{array}{l}\text { IV PCA } \\
\text { One syringe each } \\
\text { of morphine (bolus } \\
1 \mathrm{mg} \text { ), meperidine } \\
\text { (bolus } 10 \mathrm{mg} \text { ), and } \\
\text { fentanyl (bolus } \\
10 \mathrm{mg} \text { ) plus an } \\
\text { additional syringe of } \\
\text { the drug the patient } \\
\text { received first. } \\
\text { PCA given for } \\
32 \text { hours post } \\
\text { operation. }\end{array}$ & $\begin{array}{l}\text { Abdominal } \\
\text { surgery } \\
\text { (The study } \\
\text { also included } \\
\text { patients who } \\
\text { went through } \\
\text { orthopaedic } \\
\text { surgery.) }\end{array}$ & 82 & $\begin{array}{l}47 \pm 16^{* *} \\
{[\mathrm{NS}]}\end{array}$ & $\begin{array}{l}\text { No significant } \\
\text { differences in pain } \\
\text { scores were found } \\
\text { between the opioids. }\end{array}$ & $\begin{array}{l}\text { There were } \\
\text { significantly fewer } \\
\text { reports of } \\
\text { sedation in patients } \\
\text { on fentanyl versus } \\
\text { morphine }(P=.003) \text { or } \\
\text { meperidine }(P=.03) \text {. } \\
\text { No significant } \\
\text { differences in the } \\
\text { incidence of nausea, } \\
\text { vomiting, pruritus } \\
\text { were found between } \\
\text { the groups. }\end{array}$ \\
\hline $\begin{array}{l}\text { Kølliker et at } \\
\text { (1972) [28] }\end{array}$ & Pentazocine & $\begin{array}{l}\text { IV meperidine } \\
(50 \text { mg per } 70 \mathrm{~kg}) \\
\text { IV pentazocine ( } 15 \\
\text { mg per } 70 \mathrm{~kg} \text { ) } \\
\text { IV saline (placebo) } \\
\text { All patients pre- } \\
\text { medicated before } \\
\text { surgery with } 50 \mathrm{mg} \\
\text { of meperidine per } \\
70 \mathrm{~kg} \\
\text { Additional } \\
\text { pentazocine and } \\
\text { meperidine given } \\
\text { after surgery }\end{array}$ & $\begin{array}{l}\text { Upper } \\
\text { abdominal } \\
\text { surgery }\end{array}$ & 91 & $\begin{array}{l}\mathrm{NS} \\
{[\mathrm{M} / \mathrm{F}=} \\
10 / 23] \\
\mathrm{NS} \\
{[\mathrm{M} / \mathrm{F}=} \\
6 / 13] \\
\mathrm{NS} \\
{[\mathrm{M} / \mathrm{F}=} \\
16 / 23]\end{array}$ & $\begin{array}{l}\text { Meperidine had better } \\
\text { pain relief efficacy } \\
\text { than pentazocine }(P \\
<.05) \text { (investigator } \\
\text { assessment). } \\
\text { Pentazocine was an } \\
\text { effective analgesic } \\
\text { drug, but at the } \\
\text { dosage employed, it } \\
\text { was less potent and } \\
\text { had a shorter duration } \\
\text { than meperidine ( } P \\
<.05) \text {. }\end{array}$ & $\begin{array}{l}\text { More patients } \\
\text { with meperidine } \\
\text { experienced nausea. } \\
\text { a }(80 \%)\end{array}$ \\
\hline $\begin{array}{l}\text { Paymaster et } \\
\text { al (1977) [29] }\end{array}$ & $\begin{array}{l}\text { Pentazocine } \\
\text { Meptazinol }\end{array}$ & $\begin{array}{l}\text { IM meperidine } \\
(100 \mathrm{mg}) \\
\text { IM meptazinol ( } 100 \\
\mathrm{mg}) \\
\text { IM pentazocine } \\
(60 \mathrm{mg})\end{array}$ & $\begin{array}{l}\text { Abdominal } \\
\text { surgery or } \\
\text { orthopaedic } \\
\text { surgery }\end{array}$ & 75 & $\begin{array}{l}45.0 \pm 2.4 \\
{[\mathrm{M} / \mathrm{F}=} \\
3 / 22] \\
44.2 \pm 1.6 \\
{[\mathrm{M} / \mathrm{F}=} \\
3 / 22] \\
44.4 \pm 2.2 \\
{[\mathrm{M} / \mathrm{F}=} \\
3 / 22]\end{array}$ & $\begin{array}{l}\text { No differences in } \\
\text { pain relief were found } \\
\text { among the } 3 \text { drugs. }\end{array}$ & $\begin{array}{l}\text { Twelve percent of } \\
\text { the group receiving } \\
\text { pentazocine, } 28 \% \\
\text { of meptazinol, and } \\
40 \% \text { of meperidine } \\
\text { group had side } \\
\text { effects (nausea, } \\
\text { vomiting, confusion, } \\
\text { pallor, sweating), } \\
\text { but these differences } \\
\text { were not statistically } \\
\text { significant. }\end{array}$ \\
\hline
\end{tabular}


Table 1 (cont.). Randomized controlled trials of meperidine in managing pain after abdominal surgery

\begin{tabular}{|c|c|c|c|c|c|c|c|}
\hline $\begin{array}{c}\text { Author (yr) } \\
\text { [citation] }\end{array}$ & $\begin{array}{c}\text { Comparative } \\
\text { Drug }\end{array}$ & $\begin{array}{c}\text { Dose, Dosing } \\
\text { Frequency and } \\
\text { Administration } \\
\text { Routes }\end{array}$ & Surgery Type & $\begin{array}{l}\text { Number } \\
\text { of } \\
\text { Patients }\end{array}$ & $\begin{array}{c}\text { Mean } \\
\text { Age } \\
\text { [Gender] }\end{array}$ & Analgesic Results & Adverse Event \\
\hline $\begin{array}{l}\text { Carl et al } \\
\text { (1987) [30] }\end{array}$ & Buprenorphine & $\begin{array}{l}\text { IM meperidine ( } 75 \\
\text { mg per injection) } \\
\text { Sublingual } \\
\text { buprenorphine }(0.4 \\
\text { mg) } \\
\text { IM buprenorphine } \\
(0.3 \mathrm{mg})\end{array}$ & $\begin{array}{l}\text { Major } \\
\text { abdominal } \\
\text { surgery }\end{array}$ & 54 & $\begin{array}{l}58,52 \\
55 \text { of } 3 \\
\text { groups }^{* * *} \\
{[\mathrm{NS}]}\end{array}$ & $\begin{array}{l}\text { Median pain intensity } \\
\text { differences showed } \\
\text { equal pain relief in all } \\
3 \text { groups. }\end{array}$ & $\begin{array}{l}\text { Five patients on } \\
\text { meperidine had } \\
\text { respiratory acidosis; } \\
3 \text { required IPPV } \\
\text { treatment, and } \\
\text { one case in the IM } \\
\text { buprenorphine } \\
\text { group required IPPV } \\
\text { treatment. } \\
\text { Significantly more } \\
\text { patients on IM } \\
\text { meperidine had } \\
\text { nausea. }\end{array}$ \\
\hline $\begin{array}{l}\text { Patel et al } \\
\text { (1980) [31] }\end{array}$ & Dipyrone & $\begin{array}{l}\text { IM meperidine } \\
(100 \mathrm{mg}) \\
\text { IM dipyrone }(2.5 \\
\mathrm{mg})\end{array}$ & $\begin{array}{l}\text { Elective } \\
\text { exploratory } \\
\text { laparotomy }\end{array}$ & 100 & $\begin{array}{l}58,52 \\
55 \text { of } 3 \\
\text { groups }^{* * *} \\
{[\mathrm{NS}]}\end{array}$ & $\begin{array}{l}\text { Maximum pain relief } \\
\text { was seen } 2 \text { hours after } \\
\text { drug administration } \\
\text { in both groups } \\
\text { and there was no } \\
\text { statistically significant } \\
\text { difference in pain } \\
\text { scores. }\end{array}$ & $\begin{array}{l}\text { No treatment-related } \\
\text { adverse effects were } \\
\text { recorded. In the } \\
\text { meperidine group, } \\
\text { one patient had } \\
\text { rigors, and another } \\
\text { had retention of } \\
\text { urine. }\end{array}$ \\
\hline $\begin{array}{l}\text { Hew et al } \\
\text { (1987) [32] }\end{array}$ & Nalbuphine & $\begin{array}{l}\text { IM meperidine ( } 75 \\
\mathrm{mg}) \\
\text { IV nalbuphine ( } 20 \\
\mathrm{mg}) \\
\text { IV nalbuphine ( } 40 \\
\mathrm{mg}) \\
\text { Medication given } \\
\text { for } 2 \text { hours }\end{array}$ & $\begin{array}{l}\text { Abdominal } \\
\text { surgery (the } \\
\text { study also } \\
\text { included } \\
\text { patients who } \\
\text { went through } \\
\text { orthopaedic } \\
\text { surgery) }\end{array}$ & 150 & $\begin{array}{l}42 \pm 11 \\
{[\mathrm{M} / \mathrm{F}=} \\
19 / 31] \\
40 \pm 11 \\
{[\mathrm{M} / \mathrm{F}=} \\
9 / 41] \\
40 \pm 11 \\
{[\mathrm{M} / \mathrm{F}=} \\
8 / 42]\end{array}$ & $\begin{array}{l}\text { The analgesic efficacy } \\
\text { of nalbuphine (both } \\
20 \mathrm{mg} \text { and } 40 \mathrm{mg} \text { ) was } \\
\text { similar to meperidine } \\
75 \mathrm{mg} \text {. } \\
\text { No significant } \\
\text { differences were } \\
\text { found in pain } \\
\text { intensity, pain } \\
\text { intensity differences, } \\
\text { or in pain relief. }\end{array}$ & $\begin{array}{l}\text { Twenty-two percent } \\
\text { of patients on } \\
\text { meperidine and } \\
2 \%-6 \% \text { patients } \\
\text { on nalbuphine } 20 \\
\text { mg had nausea } \\
\text { and vomiting. } \\
\text { This difference } \\
\text { was statistically } \\
\text { significant. No } \\
\text { differences were } \\
\text { found between } \\
\text { nalbuphine } 40 \mathrm{mg} \\
\text { and meperidine. }\end{array}$ \\
\hline $\begin{array}{l}\text { Folsland et al } \\
\text { (1990) [33] }\end{array}$ & Ketorolac & $\begin{array}{l}\text { IM meperidine } \\
(100 \mathrm{mg}) \\
\text { IM ketorolac }(10 \\
\mathrm{mg}) \\
\text { IM ketorolac }(30 \\
\mathrm{mg}) \\
\text { IM placebo }\end{array}$ & $\begin{array}{l}\text { Major } \\
\text { abdominal } \\
\text { surgery }\end{array}$ & 129 & $\begin{array}{l}57.0 \pm 16.3 \\
{[\mathrm{M} / \mathrm{F}=} \\
16 / 11] \\
57.4 \pm 13.8 \\
{[\mathrm{M} / \mathrm{F}=} \\
7 / 25] \\
54.7 \pm 16.8 \\
{[\mathrm{M} / \mathrm{F}=} \\
17 / 12] \\
57.7 \pm 16.7 \\
{[\mathrm{M} / \mathrm{F}=} \\
20 / 12]\end{array}$ & $\begin{array}{l}\text { During the first } 2 \\
\text { hours, meperidine } \\
\text { had significantly } \\
\text { better pain relief } \\
\text { efficacy than ketorolac } \\
\text { or placebo }(P<.05) \text {. } \\
\text { At } 4-7 \text { hours after } \\
\text { drug administration, } \\
\text { there was no } \\
\text { significant difference } \\
\text { in pain scores. }\end{array}$ & $\begin{array}{l}\text { Seven patients on } 10 \\
\text { mg ketorolac, } 2 \text { on } 30 \\
\text { mg ketorolac, } 8 \text { on } \\
100 \text { mg meperidine, } \\
\text { and } 2 \text { on placebo had } \\
\text { nausea. }\end{array}$ \\
\hline
\end{tabular}


Table 1 (cont.). Randomized controlled trials of meperidine in managing pain after abdominal surgery

\begin{tabular}{|c|c|c|c|c|c|c|c|}
\hline $\begin{array}{c}\text { Author (yr) } \\
\text { [citation] }\end{array}$ & $\begin{array}{c}\text { Comparative } \\
\text { Drug }\end{array}$ & $\begin{array}{c}\text { Dose, Dosing } \\
\text { Frequency and } \\
\text { Administration } \\
\text { Routes }\end{array}$ & Surgery Type & $\begin{array}{l}\text { Number } \\
\text { of } \\
\text { Patients }\end{array}$ & $\begin{array}{c}\text { Mean } \\
\text { Age } \\
\text { [Gender] }\end{array}$ & Analgesic Results & Adverse Event \\
\hline $\begin{array}{l}\text { Petersson et } \\
\mathrm{al}(1986) \text { [34] }\end{array}$ & Physostigmine & $\begin{array}{l}\text { IV meperidine } \\
\text { chloride }(50 \mathrm{mg}) \\
\text { IV physostigmine } \\
\text { salicylate ( } 2 \mathrm{mg}) \\
\text { IV saline (placebo) }\end{array}$ & $\begin{array}{l}\text { Abdominal } \\
\text { surgery (the } \\
\text { study also } \\
\text { included } \\
\text { patients who } \\
\text { went through } \\
\text { orthopedic } \\
\text { surgery, ENT) }\end{array}$ & 60 & $\begin{array}{l}48.2 \pm 15.7 \\
{[\mathrm{M} / \mathrm{F}=} \\
5 / 15] \\
45.7 \pm 15.7 \\
{[\mathrm{M} / \mathrm{F}=} \\
3 / 17] \\
47.0 \pm 16.6 \\
{[\mathrm{M} / \mathrm{F}=} \\
3 / 17]\end{array}$ & $\begin{array}{l}\text { After } 15 \text { minutes, } \\
\text { there was no } \\
\text { difference between } \\
\text { physostigmine and } \\
\text { meperidine. } \\
\text { From } 30 \text { minutes } \\
\text { onwards, meperidine } \\
\text { had significantly } \\
\text { reduced pain scores } \\
\text { versus placebo and } \\
\text { physostigmine }(P< \\
.05) \text {. }\end{array}$ & $\begin{array}{l}\text { One patient each } \\
\text { in the meperidine } \\
\text { and placebo groups } \\
\text { and } 6 \text { patients in the } \\
\text { physostigmine group } \\
\text { had nausea. } \\
\text { No other side effects } \\
\text { related } \\
\text { to the test drugs were } \\
\text { noted. } \\
\text { The meperidine } \\
\text { group had a higher } \\
\text { level of sedation }(P \\
<.05) \text { until one hour } \\
\text { post administration. }\end{array}$ \\
\hline
\end{tabular}

Abbreviations: ENT, Ear, Nose, and Throat; F, female; IM, intramuscular; IP, intraperitoneal; IPPV, intermittent positive-pressure ventilation; IV, intravenous; M, male; NS, not specified; PCA, patient controlled analgesia; VAS, Visual Analog Scale

${ }^{*}$ Data were presented as mean (range)

${ }^{* *}$ Data were presented regardless of treatment groups

${ }^{* * *}$ Data were presented as median only and not specific to each group

dominal surgery. A study of patients who either underwent abdominal or orthopedic surgery, however, found no significant differences in pain scores between PCA meperidine and PCA morphine (27). Intravenous pentazocine was less potent than IV meperidine in patients who underwent upper abdominal surgery (28), and a study investigating the same drugs intramuscularly after abdominal surgery found no significant difference in pain relief between the 2 analgesics (29).

Other clinical studies showed that IM meperidine had similar analgesic efficacy as sublingual buprenorphine after major abdominal surgery (30), IM dipyrone after elective exploratory laporatomy (31), and IV naIbuphine (32) and IM ketorolac (33) after abdominal surgery. A study published in 1986 demonstrated that meperidine had better analgesic efficacy than IV physostigmine following major abdominal surgery (34).

\subsubsection{Labor Analgesia}

Thirteen studies compared the analgesic efficacy of IM or IV meperidine for labor pain control versus other analgesics (Table 2), and most of them reported inferior analgesic efficacy compared to meperidine (35-45) except 2 studies comparing meperidine with tramadol (46) and saline (47). A study by Keskin et al (46) showed a significantly greater reduction in labor pain after administration of meperidine compared to tramadol according to the Wong-Baker Faces Pain Rating Scale in the first 30 and 60 minutes, but similar pain control was observed afterwards. One study found meperidine plus promazine to have less effective analgesia when compared to epidural bupivacaine (35). Four other studies showed that IM meperidine had significantly inferior analgesic efficacy compared to epidural bupivacaine, remifentanil, and diamorphine for labor pain control $(36,37,40,43-45)$. Additionally, IV PCA meperidine compared to epidural bupivacaine during spontaneous labor had significantly less analgesic efficacy (38). Submucous paracervical blockade with bupivacaine provided significantly better pain relief than IM meperidine during labor (39). Two studies comparing parental meperidine with SC or intranasal fentanyl (41) and IV paracetamol (42) showed similar outcomes in labor pain control.

\subsubsection{Cesarean Section}

Seven studies reported on the use of meperidine in patients who underwent Cesarean section (Table 3). Intramuscular meperidine was as effective in controlling post-Cesarean section pain as oral methadone (48), but less effective than IM or rectal diclofenac in 3 other studies (49-51). Comparable analgesic efficacy was reported from one study comparing SC meperidine with oral diclofenac (52). Two studies showed that PCA 
Table 2. Randomized controlled trials of meperidine in managing pain after spontaneous or induced labor.

\begin{tabular}{|c|c|c|c|c|c|c|c|}
\hline 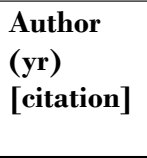 & $\begin{array}{l}\text { Comparative } \\
\text { Drug }\end{array}$ & $\begin{array}{l}\text { Dose, Dosing } \\
\text { Frequency and } \\
\text { Administration } \\
\text { Routes }\end{array}$ & $\begin{array}{l}\text { Surgery } \\
\text { Type }\end{array}$ & $\begin{array}{l}\text { Number } \\
\text { of } \\
\text { Patients }\end{array}$ & Mean Age & Analgesic Results & Adverse Event \\
\hline $\begin{array}{l}\text { Harrison } \\
\text { et al (1987) } \\
{[35]}\end{array}$ & $\begin{array}{l}\text { TENS } \\
\text { Entonox } \\
\text { Bupivacaine }\end{array}$ & $\begin{array}{l}\text { IM } 50 \text { mg } \\
\text { meperidine }+50 \\
\text { mg promazine } \\
\text { TENS } \\
\text { Entonox } \\
\text { Lumbar } \\
\text { epidural } 0.375 \% \\
\text { bupivacaine (5 } \\
\text { mL) }\end{array}$ & Labor & 170 & NS & $\begin{array}{l}\text { Eighty-eight percent } \\
\text { of patients on epidural } \\
\text { said it was fully effective; } \\
90 \% \text { on Entonox, } 96 \% \\
\text { on TENS, and } 54 \% \\
\text { on meperidine plus } \\
\text { promazine found partial } \\
\text { relief. Eighty-two percent } \\
\text { of patients on TENS } \\
\text { and } 80 \% \text { on meperidine } \\
\text { plus promazine required } \\
\text { additional analgesia. }\end{array}$ & $\begin{array}{l}\text { There was no significant } \\
\text { difference between any } \\
\text { of the groups in terms of } \\
\text { cord pH or Apgar scores } \\
\text { at } 1 \text { and } 5 \text { minutes. }\end{array}$ \\
\hline $\begin{array}{l}\text { Philipsen } \\
\text { et al (1989) } \\
{[36]}\end{array}$ & Bupivacaine & $\begin{array}{l}\text { IM meperidine } \\
(75 \mathrm{mg}) \text {. Epidural } \\
\text { bupivacaine } \\
0.375 \%(1 \mathrm{~mL} / 10 \\
\mathrm{kg})\end{array}$ & $\begin{array}{l}\text { Labor - } \\
\text { spontaneous }\end{array}$ & 112 & $\begin{array}{l}26(18-40)^{*} \\
25(19-37)^{*}\end{array}$ & $\begin{array}{l}\text { The analgesic efficacy } \\
\text { of the epidural blockade } \\
\text { was significantly better } \\
\text { than that of parenteral } \\
\text { meperidine }(P<.001) \text {. }\end{array}$ & $\begin{array}{l}\text { There were no differences } \\
\text { in neonatal cord blood } \\
\text { partial pressure of carbon } \\
\text { dioxide, pH, or Apgar } \\
\text { scores. }\end{array}$ \\
\hline $\begin{array}{l}\text { Jain et al } \\
(2003) \text { [37] }\end{array}$ & $\begin{array}{l}\text { Bupivacaine } \\
\text { with fentanyl } \\
\text { Tramadol }\end{array}$ & $\begin{array}{l}\text { IM meperidine (50 } \\
\mathrm{mg} \text { for weight } \leq 50 \\
\mathrm{~kg}, 75 \mathrm{mg} \text { for } 50-75 \\
\mathrm{~kg} \text {, and } 100 \mathrm{mg} \text { if } \\
>75 \mathrm{~kg} \text {. If a repeat } \\
\text { dose was required } \\
\text { within } 4 \text { hours, half } \\
\text { of the initial dose } \\
\text { was given, with a } \\
\text { maximum of } 200 \\
\text { mg in } 4 \text { hours, } \\
\text { or } 400 \text { mg in } 24 \\
\text { hours. Each dose } \\
\text { of meperidine was } \\
\text { combined with } \\
\text { promethazine ( } 25 \\
\text { mg). Epidural } \\
\text { bupivacaine } 0.15 \% \\
(15 \text { mg) and } \\
\text { fentanyl ( } 30 \mathrm{mg} \text { ) } \\
\text { Tramadol } 1 \mathrm{mg} / \mathrm{kg}\end{array}$ & Labor & 126 & $\begin{array}{l}24.8 \pm 2.6 \\
25.7 \pm 3.1 \\
24.1 \pm 2.8\end{array}$ & $\begin{array}{l}\text { Significantly more } \\
\text { patients on epidural } \\
\text { bupivacaine achieved } \\
\text { complete pain relief } \\
\text { versus tramadol or } \\
\text { meperidine }(P<.001) .\end{array}$ & $\begin{array}{l}\text { In the epidural group, } 40 \% \\
\text { had urinary retention and } \\
16 \% \text { had motor weakness. } \\
\text { Sedation was the only } \\
\text { side effect seen in the } \\
\text { meperidine ( } 41 \% \\
\text { intrapartum and } 66 \% \\
\text { postpartum) tramadol } \\
\text { groups ( } 9 \% \text { intra- and } \\
\text { postpartum) }(P<.01) \text {. } \\
\text { Respiratory depression was } \\
\text { seen in } 3 \text { neonates in the } \\
\text { meperidine group and } 2 \text { in } \\
\text { the tramadol group. Three } \\
\text { neonates in the meperidine } \\
\text { group and } 2 \text { in the tramadol } \\
\text { group had poor respiratory } \\
\text { efforts, which improved on } \\
\text { use of naloxone and with } \\
\text { assisted ventilation for a few } \\
\text { minutes. }\end{array}$ \\
\hline $\begin{array}{l}\text { Sharma et } \\
\text { al (1997) } \\
{[38]}\end{array}$ & $\begin{array}{l}\text { Bupivacaine } \\
\text { with fentanyl }\end{array}$ & $\begin{array}{l}\text { IV PCA } \\
\text { meperidine } \\
(50 \mathrm{mg}) \text { and } \\
\text { promethazine } \\
\text { hydrochloride } \\
\text { ( } 25 \mathrm{mg} \text { ). IV } \\
\text { PCA of } 10-\mathrm{mg} \\
\text { bolus } 10 \mathrm{minutes} \\
\text { as needed for first } \\
\text { hour, } 15 \mathrm{mg} / 10 \\
\text { minutes as needed } \\
\text { until delivery. } \\
\text { Continuous } \\
\text { epidural infusion } \\
\text { of } 0.125 \% \\
\text { bupivacaine with } \\
2 \mu \mathrm{g} / \mathrm{mL} \text { fentanyl } \\
\text { followed by an } \\
\text { initial infusion rate } \\
\text { of } 8-10 \mathrm{~mL} / \mathrm{hr}\end{array}$ & Labor & 715 & $\begin{array}{l}21.8 \pm 5 \\
22.3 \pm 5\end{array}$ & $\begin{array}{l}\text { Women who received } \\
\text { epidural bupivacaine } \\
\text { reported lower pain } \\
\text { scores during labour } \\
\text { and delivery compared } \\
\text { to patient-controlled IV } \\
\text { meperidine analgesia. }\end{array}$ & $\begin{array}{l}\text { Women who received } \\
\text { epidural analgesia had } \\
\text { a significantly higher } \\
\text { incidence of hypotension } \\
\text { compared to meperidine } \\
(P<.001) \text {. There was } \\
\text { no significant difference } \\
\text { in the incidence of } \\
\text { nausea and vomiting } \\
\text { between the } 2 \text { groups. } \\
\text { Sedation scores were } \\
\text { significantly higher with } \\
\text { IV PCA compared with } \\
\text { epidural analgesia }(P \\
<.001) \text {. Significantly } \\
\text { more neonates required } \\
\text { naloxone for depressed } \\
\text { respiration with } \\
\text { meperidine than epidural } \\
\text { analgesia }(P<05) \\
\end{array}$ \\
\hline
\end{tabular}


Table 2 (cont.). Randomized controlled trials of meperidine in managing pain after spontaneous or induced labor.

\begin{tabular}{|c|c|c|c|c|c|c|c|}
\hline $\begin{array}{l}\text { Author } \\
\text { (yr) } \\
\text { [citation] }\end{array}$ & $\begin{array}{l}\text { Comparative } \\
\text { Drug }\end{array}$ & $\begin{array}{l}\text { Dose, Dosing } \\
\text { Frequency and } \\
\text { Administration } \\
\text { Routes }\end{array}$ & $\begin{array}{l}\text { Surgery } \\
\text { Type }\end{array}$ & $\begin{array}{l}\text { Number } \\
\text { of } \\
\text { Patients }\end{array}$ & Mean Age & Analgesic Results & Adverse Event \\
\hline $\begin{array}{l}\text { Jensen et al } \\
\text { (1984) [39] }\end{array}$ & Bupivacaine & $\begin{array}{l}\text { IM meperidine } \\
(75 \mathrm{mg}) \\
\text { Submucous } \\
\text { paracervical } \\
\text { blockade } 0.25 \% \\
\text { bupivacaine (12 } \\
\mathrm{mL})\end{array}$ & Labor & 117 & NS & $\begin{array}{l}\text { Seventy-eight percent } \\
\text { of the women in the } \\
\text { paracervical blockade } \\
\text { group achieved full or } \\
\text { acceptable pain relief vs } \\
31 \% \text { in the meperidine } \\
\text { group }(P<.01) \text {. } \\
\text { Average pain relief in } \\
\text { paracervical blockade } \\
\text { group was higher than } \\
\text { meperidine at } 20,40 \text {, } \\
\text { and } 60 \text { minutes. }\end{array}$ & $\begin{array}{l}\text { Two infants in the } \\
\text { paracervical blockade } \\
\text { group and one in the } \\
\text { meperidine group } \\
\text { had transient foetal } \\
\text { bradycardia. } \\
\text { Fetal distress (umbilical } \\
\text { artery pH of } 7.15 \text { or less } \\
\text { and/or a one-minute } \\
\text { Apgar score of } 7 \text { or less) } \\
\text { was more frequent in the } \\
\text { meperidine group versus } \\
\text { paracervical blockade } \\
\text { group }(P<.05) \text {. }\end{array}$ \\
\hline $\begin{array}{l}\text { Ng et al } \\
\text { (2011) [40] }\end{array}$ & Remifentanil & $\begin{array}{l}\text { IM meperidine } \\
(50-75 \mathrm{mg}) \\
\text { IV PCA bolus of } \\
\text { Remifentanil ( } 25- \\
30 \mu \mathrm{g} \text { per bolus) }\end{array}$ & Labor & 68 & $\begin{array}{l}29 \pm 5 \\
28 \pm 5\end{array}$ & $\begin{array}{l}\text { Women who received } \\
\text { patient-controlled } \\
\text { remifentanil reported } \\
\text { lower pain scores } \\
\text { during labour and } \\
\text { delivery compared } \\
\text { to IM meperidine } \\
\text { analgesia. }\end{array}$ & $\begin{array}{l}\text { There were no } \\
\text { differences in opioid- } \\
\text { related adverse outcomes } \\
\text { between groups. Nausea, } \\
\text { vomiting, and pruritus } \\
\text { were rare, but dizziness } \\
\text { was common with both } \\
\text { treatments. }\end{array}$ \\
\hline $\begin{array}{l}\text { Fleet et al } \\
(2015) \text { [41] }\end{array}$ & Fentanyl & $\begin{array}{l}\text { IM meperidine } \\
\text { ( } 100 \mathrm{mg} / 2 \mathrm{~mL} \text {; } \\
\text { initial dose } 100 \\
\text { mg and repeated } \\
\text { dose after } 3-4 \\
\text { hours if required, } \\
\text { maximum total } \\
\text { dose of } 200 \mathrm{mg}) \\
\text { SC Fentanyl (200 } \\
\text { mcg-bolus dose; } \\
\text { after one hour } \\
\text { additional } 50 \text { mcg } \\
\text { every } 15 \text { minutes as } \\
\text { requested, up to } 650 \\
\text { mcg) IN Fentanyl } \\
(54 \text { mcg; maximum } \\
\text { hourly dose was } \\
600 \text { mcg with a } \\
\text { maximum total } \\
\text { dose of } 1200 \mathrm{mcg})\end{array}$ & Labor & 156 & $\begin{array}{l}28.6 \pm 4.7 \\
29.9 \pm 5.5 \\
29.0 \pm 6.3\end{array}$ & $\begin{array}{l}\text { There was no } \\
\text { significant difference } \\
\text { between groups for } \\
\text { reductions in pain } \\
\text { scores, but all groups } \\
\text { reported clinically } \\
\text { significant reductions } \\
\text { in pain scores (mean } \\
\text { range } 1.2-16 ; P<.001 \text { ). }\end{array}$ & $\begin{array}{l}\text { Women in fentanyl } \\
\text { groups experienced } \\
\text { less sedation }(P \leq .03), \\
\text { shorter labors by at } \\
\text { least } 2 \text { hours }(P<.05), \\
\text { and fewer difficulties } \\
\text { establishing breastfeeding } \\
(P<.01) \text {. } \\
\text { Neonates in the } \\
\text { meperidine group were } \\
\text { more likely to require } \\
\text { nursery admission }(P \\
<.02) .\end{array}$ \\
\hline $\begin{array}{l}\text { Elbohoty } \\
\text { et al (2012) } \\
{[42]}\end{array}$ & Paracetamol & $\begin{array}{l}\text { IV meperidine } \\
(50 \mathrm{mg}) \\
\text { IV paracetamol } \\
(1,000 \mathrm{mg})\end{array}$ & Labor & 52 & NS & $\begin{array}{l}\text { Both potency and } \\
\text { duration of paracetamol } \\
\text { was no less than that of } \\
\text { meperidine. }\end{array}$ & $\begin{array}{l}\text { Dizziness, blurred } \\
\text { vision and vomiting } \\
\text { were observed in } \\
64 \% \text { of women given } \\
\text { meperidine, while } \\
\text { none were reported for } \\
\text { paracetamol. } \\
\text { one-minute Apgar scores } \\
\text { were significantly lower } \\
\text { in the pethidine group. }\end{array}$ \\
\hline
\end{tabular}


Table 2 (cont.). Randomized controlled trials of meperidine in managing pain after spontaneous or induced labor.

\begin{tabular}{|c|c|c|c|c|c|c|c|}
\hline $\begin{array}{l}\text { Author } \\
\text { (yr) } \\
\text { [citation] }\end{array}$ & $\begin{array}{l}\text { Comparative } \\
\text { Drug }\end{array}$ & $\begin{array}{l}\text { Dose, Dosing } \\
\text { Frequency and } \\
\text { Administration } \\
\text { Routes }\end{array}$ & $\begin{array}{l}\text { Surgery } \\
\text { Type }\end{array}$ & $\begin{array}{l}\text { Number } \\
\text { of } \\
\text { Patients }\end{array}$ & Mean Age & Analgesic Results & Adverse Event \\
\hline $\begin{array}{l}\text { Loughnan } \\
\text { et al (2000) } \\
{[43]}\end{array}$ & Bupivacaine & $\begin{array}{l}\text { IM meperidine } \\
\text { ( } 300 \mathrm{mg}) \\
\text { Epidural } \\
\text { bupivacaine } \\
\text { (initial } \\
\text { dose } 0.25 \% \\
\text { bupivacaine in } 10- \\
15 \mathrm{~mL} \text { followed } \\
\text { by infusion } \\
\text { of } 0.125 \% \\
\text { bupivacaine at } \\
10 \text { - } 15 \mathrm{~mL} / \mathrm{hr} \text { until } \\
\text { second stage). }\end{array}$ & Labor & 614 & $\begin{array}{l}26.6 \\
(15-39)^{\star *} \\
26.9 \\
(14-42)^{\star *}\end{array}$ & $\begin{array}{l}\text { Women in the epidural } \\
\text { group were significantly } \\
\text { more likely to grade their } \\
\text { pain relief as good or } \\
\text { excellent in the first and } \\
\text { second stages }(P<.001) \text {. } \\
\text { Mode of delivery was } \\
\text { similar between groups, } \\
\text { but there was a slight but } \\
\text { insignificant decrease } \\
\text { in the normal vaginal } \\
\text { delivery rate in the } \\
\text { epidural group }(P=.9) \text {. }\end{array}$ & NS \\
\hline $\begin{array}{l}\text { Wee et al } \\
(2014)[44]\end{array}$ & Diamorphine & $\begin{array}{l}\text { IM meperidine } \\
\text { ( } 150 \mathrm{mg}) \\
\text { IM diamorphine } \\
(7.5 \mathrm{mg})\end{array}$ & Labor & 484 & $\begin{array}{l}28.7 \pm 5.6 \\
28.7 \pm 6.1\end{array}$ & $\begin{array}{l}\text { There was better pain } \\
\text { relief with diamorphine } \\
\text { at } 60 \text { minutes and } \\
\text { over the whole } \\
\text { 3-hour period with a } \\
\text { significant reduction } \\
\text { in VAS scores from } \\
\text { baseline }(P<.001) \text {. }\end{array}$ & $\begin{array}{l}\text { Women in the } \\
\text { diamorphine group } \\
\text { were more likely to have } \\
\text { hemoglobin saturation } \\
\mathrm{SpO}_{2}<97 \% \text { at } 60 \\
\text { minutes }(P=.04) \text { without } \\
\text { clinically significant } \\
\text { hypoxia. Women in } \\
\text { the diamorphine group } \\
\text { were more likely to } \\
\text { experience one or more } \\
\text { nausea events during } \\
\text { the whole 3-hour period } \\
(P=.047 \text {. There was } \\
\text { more moderate or severe } \\
\text { neonatal sedation in the } \\
\text { meperidine group } 2 \text { hours } \\
\text { after delivery }(P=.04) .\end{array}$ \\
\hline $\begin{array}{l}\text { Douma et } \\
\text { al }(2010) \\
{[45]}\end{array}$ & $\begin{array}{l}\text { Remifentanil } \\
\text { Fentanyl }\end{array}$ & $\begin{array}{l}\text { IV PCA } \\
\text { meperidine } \\
\text { ( } 49.5 \text { mg-loading } \\
\text { and 5-mg boluses } \\
\text { with a maximum } \\
\text { dose limit of } 200 \\
\text { mg) } \\
\text { IV PCA } \\
\text { remifentanil } \\
\text { ( } 40 \mu g \text {-loading } \\
\text { dose and } 40 \mu \mathrm{g} \\
\text { per bolus with } \\
\text { maximum dose } \\
\text { limit of } 1200 \mu \mathrm{g} / \\
\text { hr) } \\
\text { IV PCA fentanyl } \\
(50 \mu g \text {-loading } \\
\text { and boluses of } \\
20 \mu \mathrm{g} \text { with a } \\
\text { maximum dose } \\
\text { limit of } 240 \mu \mathrm{g} / \mathrm{hr} \text { ) }\end{array}$ & Labor & 159 & $\begin{array}{l}33.6 \pm 5.5 \\
33.1 \pm 5.0 \\
33.5 \pm 4.1\end{array}$ & $\begin{array}{l}\text { Remifentanil was } \\
\text { associated with the } \\
\text { greatest decrease } \\
\text { in pain scores, but } \\
\text { the differences were } \\
\text { significant only at } \\
\text { one hour (meperidine } \\
\text { vs. remifentanil, } P< \\
.001 \text {; remifentanil vs. } \\
\text { fentanyl, } P<.01 \text { ). } \\
\text { Overall satisfaction } \\
\text { scores were higher } \\
\text { with remifentanil, } \\
\text { but produced more } \\
\text { sedation and itching. }\end{array}$ & $\begin{array}{l}\text { More periods of } \\
\text { desaturation }\left(\mathrm{SaO}_{2}<\right. \\
95 \%) \text { were observed } \\
\text { for remifentanil and } \\
\text { fentanyl. } \\
\text { There were no significant } \\
\text { differences in fetal } \\
\text { outcome between the } 3 \\
\text { groups. }\end{array}$ \\
\hline
\end{tabular}


Postoperative Analgesic Efficacy of Meperidine

Table 2 (cont.). Randomized controlled trials of meperidine in managing pain after spontaneous or induced labor.

\begin{tabular}{|c|c|c|c|c|c|c|c|}
\hline $\begin{array}{l}\text { Author } \\
\text { (yr) } \\
\text { [citation] }\end{array}$ & $\begin{array}{l}\text { Comparative } \\
\text { Drug }\end{array}$ & $\begin{array}{l}\text { Dose, Dosing } \\
\text { Frequency and } \\
\text { Administration } \\
\text { Routes }\end{array}$ & $\begin{array}{l}\text { Surgery } \\
\text { Type }\end{array}$ & $\begin{array}{l}\text { Number } \\
\text { of } \\
\text { Patients }\end{array}$ & Mean Age & Analgesic Results & Adverse Event \\
\hline $\begin{array}{l}\text { Keskin et al } \\
(2003) \text { [46] }\end{array}$ & Tramadol & $\begin{array}{l}\text { IM meperidine } \\
(100 \mathrm{mg}) \\
\text { IM tramadol } \\
(100 \mathrm{mg})\end{array}$ & Labor & 59 & $\begin{array}{l}25.07 \\
22.43\end{array}$ & $\begin{array}{l}\text { Pain relief was greater } \\
\text { in the meperidine } \\
\text { group at } 30 \text { and } 60 \\
\text { minutes after drug } \\
\text { administration }(P<.05) \text {. }\end{array}$ & $\begin{array}{l}\text { Incidence of nausea and } \\
\text { fatigue was statistically } \\
\text { higher in the tramadol } \\
\text { group at } 60 \text { minutes ( } P \\
<.05) \text {. } \\
\text { Both groups reported } \\
\text { a significant decrease } \\
\text { in systolic and diastolic } \\
\text { blood pressure and an } \\
\text { increase in heart rate. } \\
\text { No significant differences } \\
\text { in duration of labor and } \\
\text { Apgar scores were found } \\
\text { between groups. }\end{array}$ \\
\hline $\begin{array}{l}\text { Tsui et al } \\
\text { (2004) [47] }\end{array}$ & Saline & $\begin{array}{l}\text { IM meperidine } \\
(100 \mathrm{mg}) \\
\text { IM saline } \\
\text { (placebo) } \\
(100 \mathrm{mg})\end{array}$ & Labor & 50 & $\begin{array}{l}28.4 \pm 4.4 \\
28.4 \pm 5.1\end{array}$ & $\begin{array}{l}\text { There was a significant } \\
\text { reduction in VAS pain } \\
\text { scores at } 30 \text { minutes in } \\
\text { the meperidine group }\end{array}$ & $\begin{array}{l}\text { Significantly higher } \\
\text { sedation scores were } \\
\text { observed in the } \\
\text { meperidine group than } \\
\text { in the control group at } 15 \\
\text { minutes }(P=.002) \text { and at } \\
30 \text { minutes }(P=.005) \text {. } \\
\text { Similar other maternal } \\
\text { side effects and neonatal } \\
\text { outcomes were found in } \\
\text { both groups. }\end{array}$ \\
\hline
\end{tabular}

Abbreviations: Apgar, Appearance, Pulse, Grimace, Activity, and Respiration; IM, intramuscular; IN, intranasal; IV, intravenous; NS, not specified; PCA, patient controlled analgesia; SC, subcutaneous; TENS, transcutaneous electrical nerve stimulation; VAS, Visual Analog Scale.

${ }^{*}$ Data presented as median (range).

${ }^{* *}$ Data presented as mean (range).

meperidine had less analgesic efficacy when compared to PCA and epidural morphine $(53,54)$, or PCA oxymorphone (54).

\subsubsection{Gynecological Surgery}

Nine studies investigated the analgesic effect of meperidine versus other analgesics after gynecological surgery (Table 4). Three studies found no significant difference in analgesia between meperidine and morphine when used intravenously or as PCA after elective total abdominal hysterectomy, elective gynecological surgery, or abdominal hysterectomy (55-57). One study found IM meperidine to be more effective than PCA morphine following laparoscopic ovarian cystectomy (58). PCA meperidine had similar analgesic efficacy as PCA tramadol after abdominal hysterectomy $(57,59)$, and IM meperidine provided equal analgesia when compared to sublingual buprenorphine after laparotomy (60). Parenteral meperidine was less effective for pain control versus parenteral dipyrone after gyneco- logical surgery (61), and intraperitoneal (IP) meperidine was less effective than IP bupivacaine for pain relief after laparoscopic tubal ligation (62). After postpartum episiotomy, IM meperidine provided less pain relief than IM metkephamid (63).

\subsubsection{Orthopedic Surgery}

Nine studies compared meperidine to other analgesics in patients who underwent orthopedic surgery (Table 5). Although in the first 30 minutes postsurgery meperidine produced a lower pain intensity compared to tramadol, overall similar effectiveness on the pain endpoint was noted between IV meperidine and IV tramadol after total hip or total knee replacement (64), and between IM meperidine and IM ketorolac after major orthopedic surgery (65). Intramuscular meperidine was less effective than IM diclofenac after hip replacement for arthrosis (66) and IM nalbuphine after elective surgery (67). Two studies comparing IA meperidine with IA morphine after arthroscopic meniscectomy or 
Table 3. Randomized controlled trials of meperidine in managing pain after Cesarean section.

\begin{tabular}{|c|c|c|c|c|c|c|c|}
\hline $\begin{array}{l}\text { Author (yr) } \\
\text { [citation] }\end{array}$ & $\begin{array}{l}\text { Comparative } \\
\text { Drug }\end{array}$ & $\begin{array}{l}\text { Dose, Dosing } \\
\text { Frequency and } \\
\text { Administration } \\
\text { Routes }\end{array}$ & $\begin{array}{l}\text { Surgery } \\
\text { Type }\end{array}$ & $\begin{array}{l}\text { Number } \\
\text { of } \\
\text { Patients }\end{array}$ & Mean Age & Analgesic Results & Adverse Event \\
\hline $\begin{array}{l}\text { Shahraki et al } \\
\text { (2012) [48] }\end{array}$ & Methadone & $\begin{array}{l}\text { IM meperidine ( } 0.7 \\
\mathrm{mg} / \mathrm{kg} \text { every } 6 \text { hours } \\
\text { for } 24 \text { hours) } \\
\text { Oral methadone ( } 0.7 \\
\mathrm{mg} / \mathrm{kg} \text { - every } 6 \text { hours } \\
\text { for } 24 \text { hours) } \\
\text { All patients were given } \\
\text { a single dose of IM } \\
\text { meperidine ( } 50 \mathrm{mg} \text { ) in } \\
\text { the recovery room }\end{array}$ & $\begin{array}{l}\text { Cesarean } \\
\text { section }\end{array}$ & 102 & $\begin{array}{l}27.3 \pm 4.7 \\
28.4 \pm 4.3\end{array}$ & $\begin{array}{l}\text { There were } \\
\text { no significant } \\
\text { differences in pain } \\
\text { scores between } \\
\text { groups in each } \\
\text { follow-up period. }\end{array}$ & $\begin{array}{l}\text { There was no } \\
\text { difference in terms } \\
\text { of complications. No } \\
\text { complication was } \\
\text { reported in neonates } \\
\text { of the } 2 \text { groups during } \\
\text { the first } 72 \text { hours of } \\
\text { the follow-up period. }\end{array}$ \\
\hline $\begin{array}{l}\text { Rahmanpoor } \\
\text { et al (2007) } \\
{[49]}\end{array}$ & Diclofenac & $\begin{array}{l}\text { Meperidine } 25 \mathrm{mg} \\
\text { followed by } 25 \mathrm{mg} \\
\text { every } 8 \text { hours for the } \\
\text { first } 24 \text { hours } \\
100 \mathrm{mg} \\
\text { rectal;diclofenac } \\
\text { followed by } 100 \mathrm{mg} \\
\text { diclofenac every } 8 \\
\text { hours for the first } 24 \\
\text { hours }\end{array}$ & $\begin{array}{l}\text { Cesarean } \\
\text { section }\end{array}$ & 122 & $\begin{array}{l}26 \pm 5.2 \\
25.69 \pm 5.8\end{array}$ & $\begin{array}{l}\text { VAS scores for pain } \\
\text { were significantly } \\
\text { lower for diclofenac } \\
\text { at all time points } \\
\text { (i.e., } P=.046 \text { for } \\
\text { the first } 4 \text { hours } \\
\text { post-operation; } P= \\
.005 \text { for } 20-24 \text { hours } \\
\text { after operation). }\end{array}$ & $\begin{array}{l}\text { There were no } \\
\text { differences in the } \\
\text { incidences of vomiting } \\
\text { and ileus. }\end{array}$ \\
\hline $\begin{array}{l}\text { Bozkurt et al } \\
\text { (2009) [50] }\end{array}$ & $\begin{array}{l}\text { Diclofenac } \\
\text { sodium }\end{array}$ & $\begin{array}{l}\text { Meperidine ( } 6 \text { x } 50 \\
\text { mg) } \\
\text { IM diclofenac sodium } \\
(2 \times 75 \mathrm{mg}) \\
\mathrm{IM} \text { diclofenac } \\
\text { sodium }(2 \times 75 \mathrm{mg}) \\
\text { with additional } \\
\text { breakthrough } \mathrm{IM} \\
\text { meperidine }(50 \mathrm{mg}) \text { if } \\
\text { needed }\end{array}$ & $\begin{array}{l}\text { Cesarean } \\
\text { section }\end{array}$ & 130 & $\begin{array}{l}29.6 \pm 6.02 \\
30.98 \pm 5.06 \\
29.25 \pm 5.88\end{array}$ & $\begin{array}{l}\text { Diclofenac had } \\
\text { lower mean pain } \\
\text { scores versus } \\
\text { meperidine at } \\
\text { rest and during } \\
\text { coughing }(P<.05 \\
\text { for both). }\end{array}$ & $\begin{array}{l}\text { There were no } \\
\text { statistically significant } \\
\text { differences in itching, } \\
\text { nausea, vomiting } \\
\text { and abdominal } \\
\text { distension between } \\
\text { groups. Diclofenac } \\
\text { and diclofenac plus } \\
\text { meperidine resulted } \\
\text { in significantly less } \\
\text { sedation compared to } \\
\text { meperidine }(P<.05) \text {. }\end{array}$ \\
\hline $\begin{array}{l}\text { Soroori et al } \\
\text { (2006) [51] }\end{array}$ & Diclofenac & $\begin{array}{l}\text { Rectal suppository } \\
\text { diclofenac }(100 \mathrm{mg}) \text { at } \\
\text { the end of operation } \\
\text { and } 8,16 \text {, and } 24 \text { hours } \\
\text { after the operation } \\
\mathrm{IM} \text { meperidine } 1 \\
\mathrm{mg} / \mathrm{kg} \text { at the end of } \\
\text { operation and } 8,16 \text {, } \\
\text { and } 24 \text { hours after the } \\
\text { operation }\end{array}$ & $\begin{array}{l}\text { Cesarean } \\
\text { section }\end{array}$ & 240 & $\begin{array}{l}27.2 \pm 6.5 \\
26.4 \pm 5.6\end{array}$ & $\begin{array}{l}\text { Diclofenac had } \\
\text { significantly lower } \\
\text { pain scores than } \\
\text { meperidine at } 10, \\
18 \text {, and } 26 \text { hours } \\
\text { after surgery }(P< \\
.05) \text {. }\end{array}$ & $\begin{array}{l}\text { Eleven cases of } \\
\text { dizziness occurred } \\
\text { in the meperidine } \\
\text { group. No dizziness } \\
\text { was reported in the } \\
\text { diclofenac group. } \\
\text { Sedation was } \\
\text { significantly higher in } \\
\text { the meperidine group. } \\
\text { Nausea and vomiting } \\
\text { occurred at similar } \\
\text { rates in both groups. }\end{array}$ \\
\hline $\begin{array}{l}\text { Marzida et al } \\
\text { (2009) [52] }\end{array}$ & Diclofenac & $\begin{array}{l}\text { Subcutaneous } \\
\text { meperidine }(1 \mathrm{mg} / \\
\text { kg every } 8 \text { hours and } \\
\text { additional doses made } \\
\text { available on request) } \\
\text { Oral diclofenac ( } 75 \mathrm{mg} \\
\text { twice daily) } \\
\text { All patients received } \\
2-2.5 \mathrm{~mL} \text { of } 0.5 \% \\
\text { heavy bupivacaine }\end{array}$ & $\begin{array}{l}\text { Cesarean } \\
\text { section }\end{array}$ & 40 & $\begin{array}{l}30.4 \pm 4.4 \\
31.4 \pm 5.6\end{array}$ & $\begin{array}{l}\text { Pain relief was } \\
\text { adequate and } \\
\text { comparable in both } \\
\text { groups with similar } \\
\text { mean Visual Analog } \\
\text { Scores during the } \\
\text { second and third } \\
\text { day of the study } \\
\text { period. }\end{array}$ & $\begin{array}{l}\text { The incidence of } \\
\text { nausea and vomiting } \\
\text { was similar in both } \\
\text { groups. Those in the } \\
\text { meperidine group } \\
\text { were significantly } \\
\text { more sedated than } \\
\text { those in the diclofenac } \\
\text { group }(P=.024) \text {. } \\
\text { No other adverse } \\
\text { events were recorded. }\end{array}$ \\
\hline
\end{tabular}


Postoperative Analgesic Efficacy of Meperidine

Table 3 (cont.). Randomized controlled trials of meperidine in managing pain after Cesarean section.

\begin{tabular}{|c|c|c|c|c|c|c|c|}
\hline $\begin{array}{l}\text { Author (yr) } \\
\text { [citation] }\end{array}$ & $\begin{array}{l}\text { Comparative } \\
\text { Drug }\end{array}$ & $\begin{array}{l}\text { Dose, Dosing } \\
\text { Frequency and } \\
\text { Administration } \\
\text { Routes }\end{array}$ & $\begin{array}{l}\text { Surgery } \\
\text { Type }\end{array}$ & $\begin{array}{l}\text { Number } \\
\text { of } \\
\text { Patients }\end{array}$ & Mean Age & Analgesic Results & Adverse Event \\
\hline $\begin{array}{l}\text { Cade et al } \\
\text { (1993) [53] }\end{array}$ & Morphine & $\begin{array}{l}\text { IV PCA meperidine ( } 2 \\
\text { different bolus doses: } \\
10 \mathrm{mg} \text { and } 20 \mathrm{mg} \text { ) } \\
\text { Epidural morphine } \\
\text { ( } 3.5 \mathrm{mg} \text { in } 5 \mathrm{~mL} \text { saline) }\end{array}$ & $\begin{array}{l}\text { Cesarean } \\
\text { section }\end{array}$ & 132 & NS & $\begin{array}{l}\text { Epidural morphine } \\
\text { had the greatest } \\
\text { pain relief efficacy } \\
\text { (average pain score } \\
\text { of } 1.8 \text { vs 1.9-3.4 for } \\
\text { meperidine). }\end{array}$ & $\begin{array}{l}\text { In the morphine } \\
\text { group, nausea, } \\
\text { vomiting, dizziness, } \\
\text { and pruritus were } \\
\text { significantly more } \\
\text { frequent ( } P=.003 \text {, } \\
.006, .05 \text {, and } P< \\
.001 \text {, respectively), } \\
\text { while drowsiness } \\
\text { was significantly less } \\
\text { frequent ( } P=.05) \text { than } \\
\text { in other groups. } \\
\text { Numbness was } \\
\text { uncommon and was } \\
\text { not significantly } \\
\text { different between } \\
\text { groups }(P=.22) \text {. }\end{array}$ \\
\hline $\begin{array}{l}\text { Sinatra et al } \\
\text { (1989) [54] }\end{array}$ & $\begin{array}{l}\text { Morphine } \\
\text { Oxymorphone }\end{array}$ & $\begin{array}{l}\text { IV PCA meperidine } \\
(18 \mathrm{mg}) \text { every } 8 \\
\text { minutes as needed } \\
\text { within } 24 \text { hours } \\
\text { IV PCA morphine ( } 1.8 \\
\text { mg) every } 8 \text { minutes } \\
\text { as needed within } 24 \\
\text { hours } \\
\text { IV PCA oxymorphone } \\
(0.3 \mathrm{mg}) \text { every } 8 \\
\text { minutes as needed } \\
\text { within } 24 \text { hours }\end{array}$ & $\begin{array}{l}\text { Cesarean } \\
\text { section }\end{array}$ & 75 & NS & $\begin{array}{l}\text { All patients } \\
\text { achieved an } \\
\text { excellent level of } \\
\text { analgesia at rest. } \\
\text { The percentage of } \\
\text { patients reporting } \\
\text { severe pain during } \\
\text { movement was } \\
\text { highest with } \\
\text { meperidine ( } P< \\
.05) \text {. } \\
\text { VAS scores over } 24 \\
\text { hours were similar } \\
\text { between groups. }\end{array}$ & $\begin{array}{l}\text { Oxymorphone was } \\
\text { associated with the } \\
\text { highest incidence of } \\
\text { nausea and vomiting } \\
(P<.05) \text {. } \\
\text { Morphine had } \\
\text { significantly } \\
\text { increased rates of } \\
\text { sedation compared } \\
\text { to meperidine and } \\
\text { oxymorphone }(P< \\
.05) \text {. }\end{array}$ \\
\hline
\end{tabular}

Abbreviations: IM, intramuscular; IV, intravenous; NS, not specified; PCA, patient-controlled analgesia; VAS, Visual Analog Scale.

arthroscopic anterior cruciate ligament reconstruction found IA meperidine to be less effective for pain relief compared to IA morphine $(68,69)$. Another study showed that although IA meperidine provided early analgesic efficacy, it was less durable than IA morphine after arthroscopic meniscectomy (70). One study found IA morphine, meperidine, and fentanyl to have comparable analgesia after arthroscopic knee joint surgery (71). Intraarticular meperidine had similar analgesic efficacy to IA methadone after arthroscopic meniscectomy (68), and to IA fentanyl after arthroscopic knee joint surgery or knee arthroscopic meniscectomy $(71,72)$.

\subsubsection{Cardiothoracic Surgery}

Three studies investigated the analgesic effects of meperidine in cardiothoracic surgery (Table 6). Intra- muscular meperidine had a similar analgesic efficacy to IM ketamine after thoracic surgery (73), and IV meperidine also had a similar analgesic efficacy to IV morphine and IV ketobemidone post open-heart surgery (74). The analgesic effect of PCA meperidine following openheart surgery was similar to PCA fentanyl and PCA remifentanil, and better than PCA tramadol (75).

\subsubsection{Other Types of Surgery}

Seven studies investigated the analgesic efficacy of meperidine in other surgical settings (Table 7). In patients who underwent tonsillectomy, IM meperidine showed similar efficacy to IM tilidine and rectally administered indomethacin (76). One study showed that patient-controlled epidural meperidine was less effective for pain relief compared to patient-controlled 
Table 4. Randomized controlled trials of meperidine in managing pain after gynecological surgery.

\begin{tabular}{|c|c|c|c|c|c|c|c|}
\hline $\begin{array}{c}\text { Author } \\
\text { (yr) } \\
\text { [citation] }\end{array}$ & $\begin{array}{c}\text { Comparative } \\
\text { Drug }\end{array}$ & $\begin{array}{c}\text { Dose, Dosing } \\
\text { Frequency, and } \\
\text { Administration } \\
\text { Routes } \\
\end{array}$ & Surgery Type & $\begin{array}{l}\text { Number } \\
\text { of } \\
\text { Patients }\end{array}$ & Mean Age & $\begin{array}{c}\text { Analgesic } \\
\text { Results }\end{array}$ & Adverse Event \\
\hline $\begin{array}{l}\text { Stanley et al } \\
\text { (1996) [55] }\end{array}$ & Morphine & $\begin{array}{l}\text { IV PCA meperidine } \\
\text { (bolus dose } 20 \mathrm{mg} \text { ) } \\
\text { IV PCA morphine } \\
\text { (bolus dose } 2 \mathrm{mg} \text { ) } \\
\text { Increments were given } \\
\text { every } 2-4 \text { minutes by } \\
\text { the anaesthetist until } \\
\text { pain control was judged } \\
\text { to be comfortable and } \\
\text { satisfactory by the } \\
\text { patient. }\end{array}$ & $\begin{array}{l}\text { Elective total } \\
\text { abdominal } \\
\text { hysterectomy }\end{array}$ & 40 & $\begin{array}{l}39(25-49)^{*} \\
43(20-65)\end{array}$ & $\begin{array}{l}\text { There were } \\
\text { no significant } \\
\text { differences in } \\
\text { the VAS scores } \\
\text { for pain, nausea, } \\
\text { sedation, or } \\
\text { satisfaction } \\
\text { between the } \\
\text { groups. }\end{array}$ & $\begin{array}{l}\text { There were no } \\
\text { significant differences } \\
\text { in postoperative } \\
\text { sedation, nausea, } \\
\text { and requirements } \\
\text { for anti-emetics. } \\
\text { Four patients on } \\
\text { meperidine withdrew } \\
\text { due to postoperative } \\
\text { confusion; one } \\
\text { patient on morphine } \\
\text { withdrew due to } \\
\text { intractable nausea and } \\
\text { vomiting. }\end{array}$ \\
\hline $\begin{array}{l}\text { Ezri et al } \\
\text { (2002) [56] }\end{array}$ & Morphine & $\begin{array}{l}\text { IV meperidine ( } 20 \\
\mathrm{mg}) \\
\text { IV morphine }(2 \mathrm{mg})\end{array}$ & $\begin{array}{l}\text { Gynaecologic } \\
\text { surgery }\end{array}$ & 200 & $\begin{array}{l}54(29-79)^{\star *} \\
55(28-79)^{\star *}\end{array}$ & $\begin{array}{l}\text { The pain scores } \\
\text { were similar in } \\
\text { both groups. } \\
\text { Patients receiving } \\
\text { meperidine had } \\
\text { a significantly } \\
\text { better }(P<.05) \\
\text { self-reported } \\
\text { well-being score } \\
\text { at } 15 \text { minutes } \\
\text { and } 1 \text { hour post } \\
\text { PACU admission, } \\
\text { compared to } \\
\text { patients receiving } \\
\text { morphine. }\end{array}$ & $\begin{array}{l}\text { No major } \\
\text { complications were } \\
\text { noted in either } \\
\text { group - no decreased } \\
\text { respiratory rate, } \\
\text { no episodes of } \\
\text { decreased oxygen } \\
\text { saturation below } 90 \% \text {. } \\
\text { Sedation scores were } \\
\text { similar. Significantly } \\
\text { more patients on } \\
\text { meperidine required } \\
\text { anti-emetics than } \\
\text { patients on morphine } \\
(P<.05) \text {. }\end{array}$ \\
\hline $\begin{array}{l}\text { Unlugenc } \\
\text { et al (2008) } \\
{[57]}\end{array}$ & $\begin{array}{l}\text { Morphine } \\
\text { Tramadol }\end{array}$ & $\begin{array}{l}\text { Initial dose of IV } \\
\text { meperidine ( } 1 \mathrm{mg} / \\
\mathrm{kg} \text { ) and IV PCA bolus } \\
\text { of meperidine }(0.2 \\
\mathrm{mg} / \mathrm{kg} \text { ) as needed. } \\
\text { Initial dose of IV } \\
\text { morphine }(0.1 \mathrm{mg} / \mathrm{kg}) \\
\text { and IV PCA bolus of } \\
\text { morphine }(0.02 \mathrm{mg} / \mathrm{kg}) \\
\text { as needed Initial dose } \\
\text { of IV tramadol ( } 1 \mathrm{mg} / \\
\mathrm{kg} \text { ) and IV PCA bolus } \\
\text { of tramadol }(0.2 \mathrm{mg} / \\
\mathrm{kg}) \text { as needed }\end{array}$ & $\begin{array}{l}\text { Abdominal } \\
\text { hysterectomies }\end{array}$ & 90 & NS & $\begin{array}{l}\text { There were } \\
\text { no significant } \\
\text { differences } \\
\text { among groups } \\
\text { in mean pain } \\
\text { scores at any } \\
\text { time point. }\end{array}$ & $\begin{array}{l}\text { There was no } \\
\text { significant difference } \\
\text { in the incidence of } \\
\text { side effects among } \\
\text { the groups. }\end{array}$ \\
\hline $\begin{array}{l}\text { Bayar et al } \\
\text { (2008) [58] }\end{array}$ & Morphine & $\begin{array}{l}\text { IM meperidine } \\
(1 \mathrm{mg} / \mathrm{kg})(3 \\
\text { injections on the first } \\
\text { postoperative day). } \\
\text { Initial dose of IV } \\
\text { PCA morphine } \\
(0.1 \mathrm{mg} / \mathrm{kg}) ; 1 \mathrm{mg} / \mathrm{hr} \\
\text { basal rate, } 1 \mathrm{mg} \text { bolus } \\
\text { dose }\end{array}$ & $\begin{array}{l}\text { Laparoscopic } \\
\text { ovarian } \\
\text { cystectomy }\end{array}$ & 31 & $\begin{array}{l}37.8 \pm 5.4 \\
35.4 \pm 8.3\end{array}$ & $\begin{array}{l}\text { PCA morphine } \\
\text { was associated } \\
\text { with significantly } \\
\text { higher pain } \\
\text { scores compared } \\
\text { to equivalent } \\
\text { doses of IM } \\
\text { meperidine ( } P= \\
.001) \text {. }\end{array}$ & $\begin{array}{l}\text { The IM meperidine } \\
\text { group had } \\
\text { significantly higher } \\
\text { postoperative } \\
\text { mean scores on } \\
\text { Beck's Depression } \\
\text { Inventory and Beck's } \\
\text { Anxiety Inventory ( } P \\
=.03 \text { and } P=.045, \\
\text { respectively). }\end{array}$ \\
\hline
\end{tabular}

Abbreviations: IM, intramuscular; IP, intraperitoneal; IV, intravenous; NS, not specified; PACU, post-anesthesia care unit; PCA, patient controlled analgesia; VAS, Visual Analog Scale. ${ }^{*}$ Data were presented as mean (range). ${ }^{*}$ Data were presented as median (range) 
Table 4 (cont.). Randomized controlled trials of meperidine in managing pain after gynecological surgery.

\begin{tabular}{|c|c|c|c|c|c|c|c|}
\hline $\begin{array}{c}\text { Author } \\
\text { (yr) } \\
\text { [citation] }\end{array}$ & $\begin{array}{c}\text { Comparative } \\
\text { Drug }\end{array}$ & $\begin{array}{c}\text { Dose, Dosing } \\
\text { Frequency, and } \\
\text { Administration } \\
\text { Routes }\end{array}$ & Surgery Type & $\begin{array}{c}\text { Number } \\
\text { of } \\
\text { Patients }\end{array}$ & Mean Age & $\begin{array}{c}\text { Analgesic } \\
\text { Results }\end{array}$ & Adverse Event \\
\hline $\begin{array}{l}\text { Shamim et } \\
\text { al (2006) } \\
{[59]}\end{array}$ & Tramadol & $\begin{array}{l}\text { IV PCA meperidine } \\
(10 \mathrm{mg} / \mathrm{hr} \text { continuous } \\
\text { infusion, } 5 \text {-mg bolus } \\
\text { dose). IV PCA } \\
\text { tramadol ( } 10 \mathrm{mg} / \\
\text { hr basal infusion and } \\
5 \text {-mg bolus dose). All } \\
\text { patients underwent } \\
\text { induction of } \\
\text { anaesthesia with } 1 \mathrm{mg} / \\
\mathrm{kg} \text { of meperidine }\end{array}$ & $\begin{array}{l}\text { Abdominal } \\
\text { hysterectomies }\end{array}$ & 60 & $\begin{array}{l}46.5 \pm 4.42 \\
45.86 \pm 3.94\end{array}$ & $\begin{array}{l}\text { There were } \\
\text { no significant } \\
\text { differences in pain } \\
\text { scores between } \\
\text { groups. Mean } \\
\text { drug consumption } \\
\text { was significantly } \\
\text { less with tramadol } \\
\text { than with } \\
\text { meperidine }(P< \\
.05) \text {. }\end{array}$ & $\begin{array}{l}\text { The incidences of } \\
\text { nausea and vomiting } \\
\text { were similar in both } \\
\text { groups. } \\
\text { Tramadol caused } \\
\text { significantly less } \\
\text { sedation than } \\
\text { meperidine }(P<.05)\end{array}$ \\
\hline $\begin{array}{l}\text { Moa et al } \\
\text { (1990) [60] }\end{array}$ & Buprenorphine & $\begin{array}{l}\text { IM meperidine (75 } \\
\text { mg) } \\
\text { Sublingual } \\
\text { buprenorphine } \\
(0.4 \mathrm{mg})\end{array}$ & $\begin{array}{l}\text { Lower } \\
\text { laparotomy }\end{array}$ & 96 & $\begin{array}{l}44 \pm 10 \\
44 \pm 9\end{array}$ & $\begin{array}{l}\text { There were } \\
\text { no significant } \\
\text { differences in } \\
\text { pain scores } \\
\text { between groups. }\end{array}$ & $\begin{array}{l}\text { There were no } \\
\text { significant differences } \\
\text { between the groups } \\
\text { regarding respiratory } \\
\text { depression, nausea, and } \\
\text { vomiting. Nausea was } \\
\text { frequent in both groups. }\end{array}$ \\
\hline $\begin{array}{l}\text { Bloch et al } \\
\text { (1985) [61] }\end{array}$ & Dipyrone & $\begin{array}{l}\text { IM meperidine (100 } \\
\text { mg) } \\
\text { IM dipyrone ( } 2500 \\
\text { mg) }\end{array}$ & $\begin{array}{l}\text { Abdominal } \\
\text { hysterectomies }\end{array}$ & 119 & $\begin{array}{l}40.3 \\
42.7\end{array}$ & $\begin{array}{l}\text { The meperidine } \\
\text { group had } \\
\text { higher total pain } \\
\text { severity and total } \\
\text { pain VAS scores; } \\
\text { dipyrone was } \\
\text { superior in total } \\
\text { pain relief scores. }\end{array}$ & $\begin{array}{l}\text { No serious events } \\
\text { were recorded. } \\
\text { Meperidine had } \\
\text { significantly } \\
\text { higher sedation as } \\
\text { measured by a total } \\
\text { sedation analog scale } \\
(P=.002) \text {. }\end{array}$ \\
\hline $\begin{array}{l}\text { Colbert et al } \\
(2000)[62]\end{array}$ & $\begin{array}{l}\text { Bupivacaine } \\
\text { with } \\
\text { epinephrine }\end{array}$ & $\begin{array}{l}\text { IM meperidine ( } 50 \mathrm{mg} \text { ) } \\
\text { plus IP bupivacaine. IP } \\
\text { meperidine }(50 \mathrm{mg}) \\
\text { plus IP bupivacaine. } \\
\text { Dosage of bupivacaine } \\
\text { as } 80 \mathrm{~mL} \text { of } 0.125 \% \\
\text { bupivacaine with } \\
1: 200,000 \text { epinephrine } \\
\text { IP }\end{array}$ & $\begin{array}{l}\text { Laparoscopic } \\
\text { tubal ligation }\end{array}$ & 100 & $\begin{array}{l}36.9 \pm 4.1 \\
36.6 \pm 4.1\end{array}$ & $\begin{array}{l}\text { Pain scores were } \\
\text { significantly } \\
\text { lower in the } \\
\text { IP meperidine } \\
\text { group both at } \\
\text { rest }(P<.01) \text { and } \\
\text { with movement } \\
(P<.05) \text {. }\end{array}$ & $\begin{array}{l}\text { There were } \\
\text { no significant } \\
\text { differences between } \\
\text { the nausea scores } \\
\text { and the number } \\
\text { of patients } \\
\text { who vomited } \\
\text { postoperatively in } \\
\text { the } 2 \text { groups. }\end{array}$ \\
\hline $\begin{array}{l}\text { Bloomfield } \\
\text { et al (1983) } \\
{[63]}\end{array}$ & Metkephamid & $\begin{array}{l}\text { IM meperidine ( } 100 \\
\mathrm{mg} \text { ) } \\
\text { IM metkephamid ( } 70 \\
\mathrm{mg} \text { and } 140 \mathrm{mg})\end{array}$ & $\begin{array}{l}\text { Postpartum } \\
\text { episiotomy }\end{array}$ & 59 & NS & $\begin{array}{l}\text { Patients who } \\
\text { received } 140 \mathrm{mg} \\
\text { metkephamid had } \\
\text { better summed pain } \\
\text { relief, summed pain } \\
\text { intensity differences, } \\
\text { and global pain } \\
\text { relief scores than } \\
\text { those who received } \\
100 \text { mg meperidine } \\
(P<.001, P< \\
.001 \text {, and } P< \\
.002 \text { respectively). } \\
\text { Patients who } \\
\text { received } 100 \text { mg } \\
\text { meperidine had } \\
\text { better summed pain } \\
\text { relief }(P<.002) \text { and } \\
\text { intensity difference } \\
(P<.02) \text { than those } \\
\text { who received } 70 \mathrm{mg} \\
\text { metkephamid. }\end{array}$ & $\begin{array}{l}\text { Although there } \\
\text { were patients in the } \\
\text { meperidine groups } \\
\text { who experienced } \\
\text { side effects, the } \\
\text { numbers were not } \\
\text { statistically different } \\
\text { from the placebo } \\
\text { or metkephamid } \\
\text { groups. }\end{array}$ \\
\hline
\end{tabular}


Table 5. Randomized controlled trials of meperidine in managing pain after orthopedic surgery.

\begin{tabular}{|c|c|c|c|c|c|c|c|}
\hline $\begin{array}{c}\text { Author } \\
(\mathbf{y r}) \\
\text { [citation] }\end{array}$ & $\begin{array}{c}\text { Comparative } \\
\text { Drug }\end{array}$ & $\begin{array}{c}\text { Dose, Dosing } \\
\text { Frequency, and } \\
\text { Administration } \\
\text { Routes } \\
\end{array}$ & Surgery Type & $\begin{array}{c}\text { Number } \\
\text { of } \\
\text { Patients }\end{array}$ & $\begin{array}{c}\text { Mean Age } \\
\text { [Gender] }\end{array}$ & $\begin{array}{l}\text { Analgesic } \\
\text { Results }\end{array}$ & Adverse Event \\
\hline $\begin{array}{l}\text { Tarradell } \\
\text { et al (1996) } \\
{[64]}\end{array}$ & Tramadol & $\begin{array}{l}\text { IV meperidine } \\
(100 \mathrm{mg}) \\
\text { IV tramadol }(100 \\
\mathrm{mg}) \\
\text { IV saline }\end{array}$ & $\begin{array}{l}\text { Orthopaedic } \\
\text { surgery }\end{array}$ & 48 & $\begin{array}{l}63.8 \pm 1.8 \\
68.9 \pm 1.5 \\
65.1 \pm 1.4 \\
{[\mathrm{M} / \mathrm{F}=6 / 10]} \\
\text { in all groups }\end{array}$ & $\begin{array}{l}\text { For the first } \\
30 \text { minutes, } \\
\text { meperidine } \\
\text { produced lower } \\
\text { pain intensity } \\
\text { scores than } \\
\text { tramadol }(P<.05) \text {. } \\
\text { Meperidine and } \\
\text { tramadol had a } \\
\text { similar number of } \\
\text { patients who had } \\
\text { to be rescued. Both } \\
\text { opioids produced } \\
\text { a similar degree } \\
\text { of analgesia in } \\
\text { patients who were } \\
\text { not rescued. }\end{array}$ & $\begin{array}{l}\text { There were no significant } \\
\text { differences in vomiting } \\
\text { between the meperidine } \\
\text { and tramadol groups. } \\
\text { One case of urinary } \\
\text { retention and one case of } \\
\text { hypotension were observed } \\
\text { in the meperidine group. } \\
\text { Meperidine was associated } \\
\text { with a significantly higher } \\
(P<.01) \text { level of respiratory } \\
\text { depression }\left(\mathrm{PaCO}_{2}\right) \text { from } \\
5 \text { minutes to } 1 \text { hour post } \\
\text { administration. Meperidine } \\
\text { showed a significantly } \\
\text { higher level of sedation } \\
\text { in the first } 30 \text { minutes of } \\
\text { treatment when compared } \\
\text { to tramadol }(P<.05) \text {. }\end{array}$ \\
\hline $\begin{array}{l}\text { DeAndrade } \\
\text { et al (1996) } \\
{[65]}\end{array}$ & Ketorolac & $\begin{array}{l}\text { IM meperidine } \\
(100 \mathrm{mg}) \\
\text { IM ketorolac (60 } \\
\text { mg followed by } \\
30 \mathrm{mg}) \\
\text { IM placebo } \\
\text { Doses were } \\
\text { repeated every } 2-6 \\
\text { hours as necessary } \\
\text { for } 5 \text { days, up to } \\
\text { a maximum of } \\
4 \text { doses per } 24 \\
\text { hours. }\end{array}$ & $\begin{array}{l}\text { Orthopaedic } \\
\text { surgery }\end{array}$ & 244 & $\begin{array}{l}45.3(18-87)^{*} \\
{[\mathrm{M} / \mathrm{F}=} \\
121 / 79]\end{array}$ & $\begin{array}{l}\text { The 6-hour } \\
\text { summed } \\
\text { pain intensity } \\
\text { difference and } \\
\text { total pain relief } \\
\text { scores were } \\
\text { significantly } \\
\text { higher with } \\
\text { ketorolac than } \\
\text { with meperidine } \\
\text { or placebo. } \\
\text { The mean daily } \\
\text { categorical pain } \\
\text { intensity scores } \\
\text { were comparable } \\
\text { with ketorolac } \\
\text { and meperidine, } \\
\text { and both were } \\
\text { significantly } \\
\text { superior to } \\
\text { placebo. }\end{array}$ & $\begin{array}{l}\text { Ketorolac was significantly } \\
\text { better tolerated than } \\
\text { meperidine, and the } \\
\text { number of patients } \\
\text { reporting adverse events } \\
\text { was lower with ketorolac } \\
\text { than meperidine. } \\
\text { Meperidine resulted in } \\
\text { significantly more sedation } \\
\text { compared to ketorolac or } \\
\text { placebo: } P<.001 \text { at } 1 \text { hour; } \\
P \leq .01 \text { at } 2 \text { hours. Self- } \\
\text { reports of somnolence were } \\
\text { statistically significantly } \\
\text { greater with meperidine } \\
\text { (90\%) than either ketorolac } \\
\text { (74\%) or placebo ( } 44 \%) . \\
\text { More patients who received } \\
\text { meperidine }(98 \%) \text { reported } \\
\text { at least one adverse event } \\
\text { compared with either } \\
\text { ketorolac }(86 \%) \text { or placebo } \\
\text { (70\%) (overall } P<.001 ; P \\
=.028 \text { for each pairwise } \\
\text { comparison). }\end{array}$ \\
\hline $\begin{array}{l}\text { Lindgren } \\
\text { et al (1985) } \\
{[66]}\end{array}$ & Diclofenac & $\begin{array}{l}\text { IM meperidine } \\
(50 \mathrm{mg}) \\
\text { IM diclofenac ( } 75 \\
\text { mg) } \\
\text { IM placebo } \\
\text { A second injection } \\
\text { was usually } \\
\text { administered after } \\
3.5 \text { hours. }\end{array}$ & Hip surgery & 68 & $\begin{array}{l}66 \pm 10 \\
{[\mathrm{M} / \mathrm{F}} \\
=11 / 12] \\
67 \pm 8 \\
{[\mathrm{M} / \mathrm{F}} \\
=11 / 11] \\
65 \pm 9 \\
{[\mathrm{M} / \mathrm{F}=} \\
10 / 13]\end{array}$ & $\begin{array}{l}\text { Diclofenac } \\
\text { group }(P<.001) \\
\text { had better pain } \\
\text { relief than the } \\
\text { meperidine }(P< \\
.01) \text { and placebo } \\
\text { groups. }\end{array}$ & $\begin{array}{l}\text { More patients in the } \\
\text { meperidine group } \\
\text { experienced drowsiness } \\
\text { and vomiting compared to } \\
\text { the other } 2 \text { groups. }\end{array}$ \\
\hline
\end{tabular}


Table 5. (cont.) Randomized controlled trials of meperidine in managing pain after orthopedic surgery.

\begin{tabular}{|c|c|c|c|c|c|c|c|}
\hline $\begin{array}{c}\text { Author } \\
(\mathbf{y r}) \\
\text { [citation] }\end{array}$ & $\begin{array}{c}\text { Comparative } \\
\text { Drug }\end{array}$ & $\begin{array}{c}\text { Dose, Dosing } \\
\text { Frequency, and } \\
\text { Administration } \\
\text { Routes }\end{array}$ & Surgery Type & $\begin{array}{c}\text { Number } \\
\text { of } \\
\text { Patients }\end{array}$ & $\begin{array}{c}\text { Mean Age } \\
\text { [Gender] }\end{array}$ & $\begin{array}{c}\text { Analgesic } \\
\text { Results }\end{array}$ & Adverse Event \\
\hline $\begin{array}{l}\text { Brock-Utne } \\
\text { et al (1985) } \\
{[67]}\end{array}$ & Nalbuphine & $\begin{array}{l}\text { IM meperidine } \\
(100 \mathrm{mg}) \\
\text { IM nalbuphine } \\
(20 \mathrm{mg})\end{array}$ & $\begin{array}{l}\text { Orthopaedic } \\
\text { surgery }\end{array}$ & 60 & $\begin{array}{l}39.2 \pm 4.03 \\
{[\mathrm{NS}]} \\
39.4 \pm 4.01 \\
{[\mathrm{NS}]}\end{array}$ & $\begin{array}{l}\text { VAS scores were } \\
\text { statistically lower } \\
\text { in the nalbuphine } \\
\text { group at } 3(P< \\
.02) \text { and } 6 \text { hours } \\
(P<.05) \text { for } \\
\text { nalbuphine vs } \\
\text { meperidine. }\end{array}$ & $\begin{array}{l}\text { The respiration rates in } \\
\text { the meperidine group } \\
\text { were significantly more } \\
\text { depressed } 30 \text { minutes } \\
\text { after the injection than in } \\
\text { the nalbuphine group }(P \\
<.05) \text {, but this difference } \\
\text { was not clinically relevant. } \\
\text { Nalbuphine caused less } \\
\text { depression of both systolic } \\
\text { and diastolic blood pressure } \\
\text { at both } 30 \text { and } 60 \text { minutes } \\
(P<.001) \text {.There was no } \\
\text { statistically significant } \\
\text { difference in pulse rates } \\
\text { between the } 2 \text { groups. }\end{array}$ \\
\hline $\begin{array}{l}\text { Arti et al } \\
\text { (2013) [68] }\end{array}$ & $\begin{array}{l}\text { Methadone } \\
\text { Morphine }\end{array}$ & $\begin{array}{l}\text { IA meperidine (50 } \\
\text { mg) } \\
\text { IA methadone (5 } \\
\text { mg) } \\
\text { IA morphine ( } 5 \\
\text { mg) } \\
\text { All patients } \\
\text { received } \\
\text { bupivacaine } 0.5 \% \\
+1 \text { in } 200,000 \\
\text { epinephrine }\end{array}$ & $\begin{array}{l}\text { Arthroscopic } \\
\text { knee surgery } \\
\text { - meniscectomy }\end{array}$ & 140 & NS & $\begin{array}{l}\text { Morphine in } \\
\text { comparison to } \\
\text { meperidine or } \\
\text { methadone is } \\
\text { more beneficial } \\
\text { in reducing pain } \\
\text { or analgesic need } \\
\text { when it was added } \\
\text { to bupivacaine } \\
\text { injection following } \\
\text { surgery. On the } \\
\text { first day post } \\
\text { operation, there } \\
\text { was significantly } \\
\text { less pain in } \\
\text { the morphine } \\
\text { group vs in the } \\
\text { methadone, } \\
\text { meperidine, and } \\
\text { control groups }(P \\
<.05) \text {. }\end{array}$ & Not available \\
\hline $\begin{array}{l}\text { Arti et al } \\
\text { (2011) [69] }\end{array}$ & $\begin{array}{l}\text { Methadone } \\
\text { Morphine } \\
\text { Tramadol }\end{array}$ & $\begin{array}{l}\text { IA meperidine } \\
(37.5 \mathrm{mg}) \\
\text { IA methadone (5 } \\
\text { mg) } \\
\text { IA morphine } \\
(5 \mathrm{mg}) \\
\text { IA tramadol (100 } \\
\text { mg) } \\
\text { All patients } \\
\text { received } 0.5 \% \\
\text { bupivacaine }\end{array}$ & $\begin{array}{l}\text { Arthroscopic } \\
\text { knee surgery - } \\
\text { anterior cruciate } \\
\text { ligament } \\
\text { reconstruction }\end{array}$ & 150 & $\begin{array}{l}26.8 \pm 7.8 \\
(\mathrm{M} / \mathrm{F}=23 / 7) \\
28.9 \pm 7.63 \\
(\mathrm{M} / \mathrm{F}=22 / 8) \\
31.5 \pm 5.9 \\
(\mathrm{M} / \mathrm{F}=23 / 7) \\
27.5 \pm 7.4 \\
(\mathrm{M} / \mathrm{F}=22 / 8)\end{array}$ & $\begin{array}{l}\text { Morphine had } \\
\text { better pain } \\
\text { relief efficacy } \\
\text { (VAS score } 1.7 \\
(P=.024) \text { than } \\
\text { meperidine (VAS } \\
\text { score } 1.7) \text { in the } \\
\text { first } 4 \text { hours after } \\
\text { surgery. }\end{array}$ & Not available \\
\hline
\end{tabular}


Table 5. (cont.) Randomized controlled trials of meperidine in managing pain after orthopedic surgery.

\begin{tabular}{|c|c|c|c|c|c|c|c|}
\hline $\begin{array}{c}\text { Author } \\
(\mathbf{y r}) \\
\text { [citation] }\end{array}$ & $\begin{array}{c}\text { Comparative } \\
\text { Drug }\end{array}$ & $\begin{array}{c}\text { Dose, Dosing } \\
\text { Frequency, and } \\
\text { Administration } \\
\text { Routes }\end{array}$ & Surgery Type & $\begin{array}{l}\text { Number } \\
\text { of } \\
\text { Patients }\end{array}$ & $\begin{array}{c}\text { Mean Age } \\
\text { [Gender] }\end{array}$ & $\begin{array}{l}\text { Analgesic } \\
\text { Results }\end{array}$ & Adverse Event \\
\hline $\begin{array}{l}\text { Lyons et al } \\
\text { (1995) [70] }\end{array}$ & Morphine & $\begin{array}{l}\text { IA meperidine ( } 50 \\
\text { mg) } \\
\text { IA morphine } \\
(5 \mathrm{mg}) \\
\text { IA saline (placebo) } \\
\text { All patients } \\
\text { received standard } \\
\text { postoperative } \\
\text { analgesia (bolus } \\
\text { doses of IV } \\
\text { meperidine } 25 \mathrm{mg} \\
\text { in the recovery } \\
\text { room or } \\
\text { mefenamic acid } \\
500 \text { mg orally on } \\
\text { request in } \\
\text { the ward). } \\
\text { Patients were } \\
\text { discharged with } \\
\text { mefenamic acid } \\
\text { to take } 6 \text { hourly as } \\
\text { required. }\end{array}$ & $\begin{array}{l}\text { Arthroscopic } \\
\text { knee surgery }\end{array}$ & 60 & $\begin{array}{l}32.1 \\
(18-57)^{\star \star} \\
(\mathrm{M} / \mathrm{F}=15 / 5) \\
29.5 \\
(18-48)^{\star \star} \\
(\mathrm{M} / \mathrm{F}=17 / 3) \\
32.1 \\
(18-57)^{\star \star} \\
(\mathrm{M} / \mathrm{F}=15 / 5)\end{array}$ & $\begin{array}{l}\text { Both treatment } \\
\text { groups had } \\
\text { significantly } \\
\text { lower pain scores } \\
\text { compared with } \\
\text { placebo. } \\
\text { Patients in the } \\
\text { meperidine group } \\
\text { had significantly } \\
\text { lower pain scores } \\
\text { than the morphine } \\
\text { group at } 0.5 \text {, } \\
1 \text {, and } 2 \text { hours } \\
\text { post operation } \\
(P<.05) \text {, but } \\
\text { significantly } \\
\text { higher scores at } 12 \\
\text { and } 24 \text { hours }(P \\
<.05) \text {. }\end{array}$ & Not available \\
\hline $\begin{array}{l}\text { Söderlund } \\
\text { et al (1997) } \\
{[71]}\end{array}$ & $\begin{array}{l}\text { Morphine } \\
\text { Fentanyl }\end{array}$ & $\begin{array}{l}\text { IA/IM meperidine } \\
(10 \mathrm{mg}) \\
\text { IA/IM morphine } \\
(1 \mathrm{mg}) \\
\text { IA/IM fentanyl } \\
(10 \mu \mathrm{g})\end{array}$ & $\begin{array}{l}\text { Arthroscopic } \\
\text { knee surgery }\end{array}$ & 70 & $\begin{array}{l}26.8 \pm 7.8 \\
(\mathrm{M} / \mathrm{F}=23 / 7)\end{array}$ & $\begin{array}{l}\text { There were } \\
\text { no significant } \\
\text { differences in } \\
\text { postoperative pain } \\
\text { intensity or need } \\
\text { for analgesics. }\end{array}$ & $\begin{array}{l}\text { All patients could be } \\
\text { discharged from the } \\
\text { hospitals as normal, and } \\
\text { no specific side effects } \\
\text { were recorded during the } \\
\text { first } 24 \text { hours. }\end{array}$ \\
\hline $\begin{array}{l}\text { Saryazdi et } \\
\text { al (2006) } \\
{[72]}\end{array}$ & $\begin{array}{l}\text { Fentanyl } \\
\text { Dexamethasone }\end{array}$ & $\begin{array}{l}\text { IA meperidine }(20 \\
\mathrm{mg}) \\
\text { IA fentanyl }(50 \mu \mathrm{g}) \\
\text { IA dexamethasone } \\
(8 \mathrm{mg})\end{array}$ & $\begin{array}{l}\text { Arthroscopic } \\
\text { knee surgery }\end{array}$ & 48 & $\begin{array}{l}26 \pm 8 \\
24.7 \pm 6.01 \\
26 \pm 4.9 \\
\text { [All patients } \\
\text { are male in } \\
\text { this study] }\end{array}$ & $\begin{array}{l}\text { Better } \\
\text { postoperative } \\
\text { analgesia, lower } \\
\text { pain scores, and } \\
\text { shorter time-to- } \\
\text { walk were found } \\
\text { with fentanyl } \\
\text { and meperidine } \\
\text { compared to } \\
\text { dexamethasone, } \\
\text { but the results } \\
\text { were not } \\
\text { significantly } \\
\text { different between } \\
\text { the fentanyl } \\
\text { and meperidine } \\
\text { groups. }\end{array}$ & Not available \\
\hline
\end{tabular}

Abbreviations: IA, intraarticular; IM, intramuscular; IV, intravenous; NS, not specified; $\mathrm{PaCO}_{2}$, partial pressure of carbon dioxide; PCA, patient controlled analgesia; VAS, Visual Analog Scale; M, male; F, female

${ }^{*}$ Data were presented as mean (range) for patients evaluable for efficacy analysis regardless of treatment groups

${ }^{*}$ Data were presented as mean (range) 
Postoperative Analgesic Efficacy of Meperidine

Table 6. Randomized controlled trials of meperidine in managing pain after cardiothoracic surgery

\begin{tabular}{|c|c|c|c|c|c|c|c|}
\hline $\begin{array}{c}\text { Author } \\
(\mathbf{y r}) \\
\text { [citation] }\end{array}$ & $\begin{array}{c}\text { Comparative } \\
\text { Drug }\end{array}$ & $\begin{array}{c}\text { Dose, Dosing } \\
\text { Frequency, and } \\
\text { Administration routes }\end{array}$ & $\begin{array}{c}\text { Surgery } \\
\text { Type }\end{array}$ & $\begin{array}{l}\text { Number } \\
\text { of } \\
\text { Patients }\end{array}$ & $\begin{array}{c}\text { Mean Age } \\
\text { [Gender] }\end{array}$ & $\begin{array}{c}\text { Analgesic } \\
\text { Results }\end{array}$ & Adverse Event \\
\hline $\begin{array}{l}\text { Dich- } \\
\text { Nielsen et } \\
\text { al (1992) } \\
{[73]}\end{array}$ & Ketamine & $\begin{array}{l}\text { One IM injection of } \\
\text { meperidine } \\
(1 \mathrm{mg} / \mathrm{kg}) \\
\text { One IM injection of } \\
\text { ketamine }(1 \mathrm{mg} / \mathrm{kg}) \\
\text { Injections were repeated if } \\
\text { the effect declined }\end{array}$ & $\begin{array}{l}\text { Pulmonary } \\
\text { surgery }\end{array}$ & 30 & $\begin{array}{l}64(31-73)^{*} \\
{[N S]} \\
61(28-73)^{*} \\
{[N S]}\end{array}$ & $\begin{array}{l}\text { There was no } \\
\text { significant } \\
\text { difference } \\
\text { between the } \\
\text { analgesic } \\
\text { effects of } \\
\text { ketamine and } \\
\text { meperidine. }\end{array}$ & $\begin{array}{l}\text { The incidence of } \\
\text { adverse reactions } \\
\text { was low and not } \\
\text { significantly different } \\
\text { between the groups. } \\
\text { Meperidine was } \\
\text { associated with a } \\
\text { significantly higher } \\
(P<.05) \text { level of } \\
\text { respiratory depression } \\
\left(\mathrm{PaCO}_{2}\right) \text { from } 30 \\
\text { minutes to } 3 \text { hours post } \\
\text { administration. }\end{array}$ \\
\hline $\begin{array}{l}\text { Ohqvist et } \\
\text { al (1991) } \\
{[74]}\end{array}$ & $\begin{array}{l}\text { Morphine } \\
\text { Ketobemidone }\end{array}$ & $\begin{array}{l}\text { IV morphine }(0.5 \mathrm{mg} / \mathrm{mL}) \\
\text { IV meperidine }(5 \mathrm{mg} / \mathrm{mL}) \\
\text { IV ketobemidone }(0.375 \\
\mathrm{mg} / \mathrm{mL}) \\
\text { Continuous infusions were } \\
\text { applied }\end{array}$ & $\begin{array}{l}\text { Open-heart } \\
\text { surgery }\end{array}$ & 81 & $\begin{array}{l}63 \pm 1.6 \\
{[\mathrm{M} / \mathrm{F}=20 / 7]} \\
61 \pm 2.3 \\
{[\mathrm{M} / \mathrm{F}=22 / 4]} \\
61 \pm 1.8 \\
{[\mathrm{M} / \mathrm{F}=20 / 8]}\end{array}$ & $\begin{array}{l}\text { There were } \\
\text { no significant } \\
\text { differences } \\
\text { in pain relief } \\
\text { between the } 3 \\
\text { analgesics. }\end{array}$ & $\begin{array}{l}\text { There were no } \\
\text { significant differences } \\
\text { in side effects like } \\
\text { shivering, nausea, or } \\
\text { vomiting between the } 3 \\
\text { analgesics. }\end{array}$ \\
\hline $\begin{array}{l}\text { Oztekin et } \\
\text { al (2006) } \\
{[75]}\end{array}$ & $\begin{array}{l}\text { Morphine } \\
\text { Fentanyl } \\
\text { Remifentanil } \\
\text { Tramadol }\end{array}$ & $\begin{array}{l}\text { Meperidine: } 0.7 \mathrm{mg} / \mathrm{kg} / \mathrm{hr} \text { - } \\
\text { IV infusion dose; } \\
10 \mathrm{mg} / \mathrm{kg} / \mathrm{hr} \text {-bolus dose } \\
\text { Morphine: } 0.1 \mathrm{mg} / \mathrm{kg} / \mathrm{hr} \text {-IV } \\
\text { infusion dose; } \\
1 \mathrm{mg} / \mathrm{kg} / \mathrm{hr} \text {-bolus dose } \\
\text { Fentanyl: } 0.3 \mathrm{mcg} / \mathrm{kg} / \mathrm{hr}-\mathrm{IV} \\
\text { infusion dose; } \\
25 \mathrm{mcg} / \mathrm{kg} / \mathrm{hr}-\text { bolus dose } \\
\text { Remifentanil: } 0.6 \mathrm{mcg} / \mathrm{kg} / \\
\mathrm{hr}-\mathrm{IV} \text { infusion dose; } \\
10 \mathrm{mcg} / \mathrm{kg} / \mathrm{hr} \text {-bolus dose } \\
\\
\text { Tramadol: } \\
0.07 \mathrm{mg} / \mathrm{kg} / \mathrm{hr}-\mathrm{IV} \text { infusion } \\
\text { dose; } \\
10 \mathrm{mg} / \mathrm{kg} / \mathrm{hr} \text {-bolus dose } \\
\text { IV PCA with continuous } \\
\text { background infusion }\end{array}$ & $\begin{array}{l}\text { Open heart } \\
\text { surgery with } \\
\text { sternotomy }\end{array}$ & 50 & $\begin{array}{l}49 \pm 15.18 \\
{[\mathrm{M} / \mathrm{F}=8 / 2]} \\
58.8 \pm 7.96 \\
{[\mathrm{M} / \mathrm{F}=8 / 2]} \\
55.1 \pm 6.98 \\
{[\mathrm{M} / \mathrm{F}=6 / 4]} \\
57.12 \pm \\
11.99 \\
{[\mathrm{M} / \mathrm{F}=7 / 3]} \\
55.33 \pm \\
12.75 \\
{[\mathrm{M} / \mathrm{F}=7 / 3]}\end{array}$ & $\begin{array}{l}\text { Tramadol had } \\
\text { significantly } \\
\text { higher VAS } \\
\text { score }(4 ; P \\
=.001) \text { vs } \\
\text { meperidine } \\
\text { (3) at } 24 \\
\text { hours post- } \\
\text { operation. } \\
\text { Patients } \\
\text { in the } \\
\text { meperidine } \\
\text { group } \\
\text { required } \\
\text { the lowest } \\
\text { number of } \\
\text { additional } \\
\text { bolus doses } \\
(P=.001) .\end{array}$ & $\begin{array}{l}\text { Mean levels of minute } \\
\text { ventilation, heart } \\
\text { rate, } \mathrm{PaCO}_{2} \text {, oxygen } \\
\text { saturation, and SBP } \\
\text { after } 24 \text { hours were not } \\
\text { significantly different } \\
\text { between groups. } \\
\text { Remifentanil and } \\
\text { meperidine had fewer } \\
\text { side effects than the } \\
\text { other } 3 \text { drugs. }\end{array}$ \\
\hline
\end{tabular}

Abbreviations: F, female; IM, intramuscular; IV, intravenous; M, male; MMCHS, morphine-soaked microfibrillar collagen hemostatic sponge on the surface of the dural sac; NS, not specified; $\mathrm{PaCO}_{2}$, arterial partial pressure of carbon dioxide; PCA, patient-controlled analgesia; PCEA, patientcontrolled epidural analgesia; PRN, as needed; VAS, Visual Analog Scale

${ }^{\star}$ Data were presented regardless of treatment groups

${ }^{*}$ Data were presented as sex ratio

${ }^{* * *}$ Data were presented as mean (range)

epidural fentanyl after hypospadias surgery (77). A study in urological surgery observed better pain relief using IV meperidine when compared to IV tramadol (78). In a study involving patients who underwent oral surgery, IM meperidine was less efficacious than IM ketorolac (79). One other study found IM meperidine to be superior to diclofenac suppository for laminectomy (80). Finally, IM meperidine had a comparable analgesic effect to transnasal butorphanol for anal fistulotomy (81).

\subsection{Side Effects and Safety Outcomes}

All included studies revealed at least one opioidrelated adverse effect of meperidine including nausea, vomiting, sedation, pruritus, alterations in cardiopulmonary function, dry mouth, shivering, sweating, 
Table 7. Randomized controlled trials of meperidine in managing pain after cardiothoracic surgery

\begin{tabular}{|c|c|c|c|c|c|c|c|}
\hline $\begin{array}{c}\text { Author (yr) } \\
\text { [citation] }\end{array}$ & $\begin{array}{c}\text { Comparative } \\
\text { Drug }\end{array}$ & $\begin{array}{c}\text { Dose, Dosing } \\
\text { Frequency, and } \\
\text { Administration } \\
\text { Routes }\end{array}$ & $\begin{array}{c}\text { Surgery } \\
\text { Type }\end{array}$ & $\begin{array}{c}\text { Number } \\
\text { of } \\
\text { Patients }\end{array}$ & $\begin{array}{l}\text { Mean Age } \\
\text { [Gender] }\end{array}$ & $\begin{array}{l}\text { Analgesic } \\
\text { Results }\end{array}$ & Adverse Event \\
\hline $\begin{array}{l}\text { Saarnivaara } \\
\text { et al (1980) } \\
\text { [76] }\end{array}$ & $\begin{array}{l}\text { Tilidine } \\
\text { Indomethacin }\end{array}$ & $\begin{array}{l}\text { IM meperidine } 1 \\
\mathrm{mg} / \mathrm{kg} \\
\mathrm{IM} \text { tilidine } 2.5 \mathrm{mg} / \mathrm{kg} \\
\text { Indomethacin } 100 \\
\text { mg suppository }\end{array}$ & Tonsillectomy & 87 & $\begin{array}{l}25.5 \pm 8.8 \\
{[\mathrm{M} / \mathrm{F}=11 / 18]} \\
24.7 \pm 9.9 \\
{[\mathrm{M} / \mathrm{F}=8 / 21]} \\
26.5 \pm 11.0 \\
{[\mathrm{M} / \mathrm{F}=8 / 21]}\end{array}$ & $\begin{array}{l}\text { No significant } \\
\text { differences in the } \\
\text { analgesic efficacies } \\
\text { of meperidine, } \\
\text { tilidine, and } \\
\text { indomethacin. }\end{array}$ & $\begin{array}{l}\text { Bleeding from } \\
\text { the operative } \\
\text { site occurred } \\
\text { significantly } \\
\text { more in the } \\
\text { tilidine }(24 \%) \text { and } \\
\text { indomethacin } \\
(28 \%) \text { vs } \\
\text { meperidine ( } 4 \%) \\
\text { groups. }\end{array}$ \\
\hline $\begin{array}{l}\text { Sengezer et al } \\
(2002) \text { [77] }\end{array}$ & & $\begin{array}{l}\text { Loading dose: } 5 \mathrm{~mL} \\
\text { bupivacaine }(0.125 \%) \\
\text { plus } 50 \mu \mathrm{g} \text { fentanyl } \\
\text { in } 10 \mathrm{~mL} \text { saline. } \\
\text { Continuous infusion: } \\
2 \mu \mathrm{g} \text { fentanyl mixed } \\
0.125 \% \text { bupivacaine } \\
\text { in } 1 \mathrm{~mL} \text { saline } \\
\text { solution at } 3 \mathrm{~mL} / \\
\mathrm{hr} . \text { PCEA: } 5 \mathrm{~mL} \\
2 \mu \mathrm{g} \text { fentanyl mixed } \\
0.125 \% \text { bupivacaine } \\
\text { in } 1 \mathrm{~mL} \text { saline. } 1 \mathrm{mg} / \\
\mathrm{kg} \text { IM meperidine } \\
\text { followed by } 1 \mathrm{mg} / \mathrm{kg} \\
\text { meperidine every } 4 \\
\text { hours PRN }\end{array}$ & $\begin{array}{l}\text { Hypospadias } \\
\text { surgery }\end{array}$ & 20 & $\begin{array}{l}18.3 \pm 1.2 \\
\text { All are male } \\
\text { patients. }\end{array}$ & $\begin{array}{l}\text { Bupivacaine } \\
\text { plus fentanyl } \\
\text { had significantly } \\
\text { lower VAS } \\
\text { pain scores } \\
\text { compared to IM } \\
\text { meperidine on } \\
\text { days } 1 \text { through } 6 \\
(P<.05) \text {. }\end{array}$ & $\begin{array}{l}\text { No significant } \\
\text { differences in } \\
\text { side effects were } \\
\text { reported. }\end{array}$ \\
\hline $\begin{array}{l}\text { Mojtahedzadeh } \\
\text { et al (2004) [78] }\end{array}$ & Tramadol & $\begin{array}{l}\text { Bolus: } 100 \mathrm{mg}-\mathrm{IV} \\
\text { tramadol } \\
\text { PCA: IV } 50 \mathrm{mg} \\
\text { tramadol } \\
\text { Bolus: } 50 \mathrm{mg} \mathrm{IV} \\
\text { meperidine } \\
\text { PCA: IV } 25 \mathrm{mg} \\
\text { meperidine }\end{array}$ & $\begin{array}{l}\text { Urological } \\
\text { surgery }\end{array}$ & 60 & $\begin{array}{l}44.63 \pm 19.97 \\
{[\mathrm{M}: \mathrm{F}=} \\
1: 0.3]^{\star \star} \\
45.46 \pm 18.10 \\
{[\mathrm{M}: \mathrm{F}=} \\
1: 0.4]^{\star *}\end{array}$ & $\begin{array}{l}\text { No significant } \\
\text { differences } \\
\text { between tramadol } \\
\text { and meperidine } \\
\text { in the first } 7 \text { hours } \\
\text { post surgery. } \\
\text { Meperidine } \\
\text { provided superior } \\
\text { analgesia at } 8,12 \text {, } \\
\text { and } 16 \text { hours post } \\
\text { surgery }(P<.05) \text {. }\end{array}$ & $\begin{array}{l}\text { The } \mathrm{PaCO}_{2} \text { at } 1 \\
\text { and } 4 \text { hours post } \\
\text { surgery showed } \\
\text { greater } \mathrm{PaCO}_{2} \\
\text { retention }(P<.001) \\
\text { with meperidine. } \\
\text { Meperidine } \\
\text { associated } \\
\text { with greater } \\
\text { drowsiness }(P< \\
.001) \text {. Tramadol } \\
\text { associated with } \\
\text { increased sweating } \\
(P<.01) \text { and IV } \\
\text { metoclopramide } \\
\text { used to treat nausea } \\
(P<.05) .\end{array}$ \\
\hline
\end{tabular}


Table 7 (cont.). Randomized controlled trials of meperidine in managing pain after cardiothoracic surgery

\begin{tabular}{|c|c|c|c|c|c|c|c|}
\hline $\begin{array}{c}\text { Author (yr) } \\
\text { [citation] }\end{array}$ & $\begin{array}{c}\text { Comparative } \\
\text { Drug }\end{array}$ & $\begin{array}{c}\text { Dose, Dosing } \\
\text { Frequency, and } \\
\text { Administration } \\
\text { Routes }\end{array}$ & $\begin{array}{c}\text { Surgery } \\
\text { Type }\end{array}$ & $\begin{array}{c}\text { Number } \\
\text { of } \\
\text { Patients }\end{array}$ & $\begin{array}{c}\text { Mean Age } \\
\text { [Gender] }\end{array}$ & $\begin{array}{c}\text { Analgesic } \\
\text { Results }\end{array}$ & Adverse Event \\
\hline $\begin{array}{l}\text { Fricke et al } \\
\text { (1992) [79] }\end{array}$ & Ketorolac & $\begin{array}{l}\text { IM ketorolac } 10 \mathrm{mg} \\
\text { IM ketorolac } 30 \mathrm{mg} \\
\text { IM ketorolac } 90 \mathrm{mg} \\
\text { IM meperidine } 50 \\
\mathrm{mg} \\
\text { IM meperidine } 100 \\
\mathrm{mg}\end{array}$ & Oral surgery & 145 & $\begin{array}{l}23.0 \\
(15-35)^{* * *} \\
{[\mathrm{M} / \mathrm{F}=} \\
21 / 19] \\
24.3 \\
(16-46)^{* * *} \\
{[\mathrm{M} / \mathrm{F}=} \\
22 / 18] \\
23.9 \\
(17-37)^{* * *} \\
{[\mathrm{M} / \mathrm{F}=} \\
18 / 22] \\
23.9 \\
(16-35)^{* * *} \\
{[\mathrm{M} / \mathrm{F}=} \\
17 / 22] \\
23.0 \\
(17-36)^{* * *} \\
{[\mathrm{M} / \mathrm{F}=} \\
20 / 20]\end{array}$ & $\begin{array}{l}\text { Thirty mg and } 90 \\
\text { mg IM ketorolac } \\
\text { had a similar } \\
\text { efficacy profile. } \\
\text { Thirty mg IM } \\
\text { ketorolac was } \\
\text { significantly } \\
\text { better than } 10 \\
\text { mg IM ketorolac, } \\
50 \text { mg or } 100 \mathrm{mg} \\
\text { IM meperidine } \\
(P<.05) \text {. }\end{array}$ & $\begin{array}{l}\text { Patients treated } \\
\text { with } 100 \text { mg } \\
\text { IM meperidine } \\
\text { significantly more } \\
\text { drowsy compared } \\
\text { to those treated with } \\
\text { any doses of ketorolac } \\
\text { at } 30 \text { minutes and } \\
\text { one hour post dosing } \\
\text { ( } P<.05 \text { ) and more } \\
\text { drowsy at } 2 \text {-hour } \\
\text { mark compared to } \\
10 \text { mg and } 90 \text { mg } \\
\text { IM ketorolac ( } P< \\
.05) \text {. Significantly } \\
\text { more in the } \\
\text { meperidine groups } \\
\text { reported adverse } \\
\text { events compared to } \\
\text { ketorolac groups }(P< \\
.05) \text {. Nervous system- } \\
\text { related side effects } \\
\text { (vertigo, dizziness) } \\
\text { twice as common in } \\
\text { meperidine groups. } \\
\text { More pallor in } \\
\text { meperidine groups } \\
\text { (33\% each) vs } \\
\text { ketorolac groups }(3 \% \text { - } \\
10 \%) \text {. Twenty percent } \\
\text { of meperidine- } \\
\text { receiving patients } \\
\text { had digestive system } \\
\text { adverse events vs } \\
3 \% \text { when providing } \\
\text { ketorolac.Vomiting } \\
\text { occurred in } 6 \% \text { vs } 1 \% \\
\text { in the meperidine vs } \\
\text { ketorolac groups. }\end{array}$ \\
\hline $\begin{array}{l}\text { Emamhadi } \\
\text { et al (2008) } \\
{[80]}\end{array}$ & Diclofenac & $\begin{array}{l}\text { IM meperidine } 0.5 \\
\text { mg/kg every } 8 \text { hours } \\
100 \text { mg diclofenac } \\
\text { suppository every } 8 \\
\text { hours }\end{array}$ & Laminectomy & 100 & $\begin{array}{l}\mathrm{NS} \\
{[\mathrm{M} / \mathrm{F}=23 / 27]} \\
\mathrm{NS} \\
{[\mathrm{M} / \mathrm{F}=27 / 23]}\end{array}$ & $\begin{array}{l}\text { Meperidine } \\
\text { resulted in a } \\
\text { significantly } \\
\text { lower VAS pain } \\
\text { score }(P<.05) .\end{array}$ & $\begin{array}{l}\text { There were } \\
\text { no significant } \\
\text { differences in side } \\
\text { effects. }\end{array}$ \\
\hline $\begin{array}{l}\text { Mai et al } \\
\text { (2009) [81] }\end{array}$ & Butorphanol & $\begin{array}{l}\text { Transnasal } \\
\text { butorphanol } 1 \mathrm{mg} \\
\text { every } 4 \text { hours } \\
\text { IM meperidine } 0.8 \\
\text { mg/kg every } 4 \text { hours }\end{array}$ & Fistulectomy & 60 & $\begin{array}{l}37.7 \pm 10.76 \\
{[\mathrm{M} / \mathrm{F}=} \\
20 / 10] \\
38.9 \pm 10.89 \\
{[\mathrm{M} / \mathrm{F}=22 / 8]}\end{array}$ & $\begin{array}{l}\text { Pain VAS scores } \\
\text { were similar in } \\
\text { both groups. }\end{array}$ & $\begin{array}{l}\text { There were } \\
\text { no significant } \\
\text { differences in side } \\
\text { effects. }\end{array}$ \\
\hline
\end{tabular}

Abbreviations: F, female; IM, intramuscular; IV, intravenous; M, male; MMCHS, morphine-soaked microfibrillar collagen hemostatic sponge on the surface of the dural sac; NS, not specified; PaCO2, arterial partial pressure of carbon dioxide; PCA, patient-controlled analgesia; PCEA, patient-controlled epidural analgesia; PRN, as needed; VAS, Visual Analog Scale

${ }^{*}$ Data were presented regardless of treatment groups

${ }^{* *}$ Data were presented as sex ratio

${ }^{* * *}$ Data were presented as mean (range) 
dizziness, light-headedness, headache, postoperative confusion, mood changes, hallucinations, and myoclonic jerks. None of the included studies reported on addiction or abuse potential of meperidine, euphoria, serotonin syndrome, or central nervous system toxicity. Adverse events reported in most of the studies did not include a statistical analysis of the safety parameters. Moreover, the reporting methods varied. Depending on the data availability, the safety profile of meperidine in this study is reported as causing more, equal, or less of a specific adverse effect than the comparator.

\subsubsection{Meperidine versus Morphine}

Morphine was the most common comparator of meperidine. Intravenous or PCA meperidine was associated with a higher incidence of vomiting (55-57) and similar level of sedation $(27,55,56,75)$ compared to IV, PCA, or epidural morphine. One study reported PCA morphine to cause more sedation when compared to PCA meperidine after Cesarean section (54). In another study comparing IM meperidine with PCA morphine after laparoscopic ovarian cystectomy, patients receiving IM meperidine experienced more anxiety and depressive mood changes (58). Two studies compared PCA morphine with PCA or IM meperidine. The first involved postvertical gastroplasty and the second consisted of patients who either underwent orthopedic or abdominal surgery. Both studies found no difference in the occurrence of nausea, vomiting, and pruritus, despite the lower morphine equi-analgesic doses of meperidine $(25,27)$. Other studies reported no significant differences in nausea, sedation, pruritus, and shivering between equi-analgesic PCA doses of morphine and meperidine after gynecological surgeries $(55,57)$. One study found that PCA meperidine was associated with less nausea, vomiting, and headache than PCA morphine in patients who underwent open heart surgery (75).

\subsubsection{Meperidine versus Tramadol}

Three studies compared PCA or IV meperidine with PCA or IV tramadol in patients after total abdominal hysterectomies, orthopedic surgery, or urological surgery. In these studies, PCA or IV meperidine was associated with more sedation $(59,64,78)$, increased respiratory depression, and higher carbon dioxide retention than PCA and IV tramadol $(64,78)$. Intravenous tramadol resulted in higher rates of nausea compared to IV meperidine for urological surgery (78). One study reported no significant difference in the rates of vomiting between IV tramadol and IV meperidine for ortho- pedic surgery (64). Another study found no difference in the incidences of shivering, pruritus, and nausea when comparing PCA meperidine with PCA tramadol after abdominal hysterectomy (57).

\subsubsection{Meperidine versus Other Opioids}

PCA meperidine was associated with more sedation compared to PCA fentanyl after orthopedic or abdominal surgery but did not differ for nausea, vomiting, or pruritus (27). A study comparing PCA fentanyl with PCA meperidine after open heart surgery found higher levels of sedation with meperidine (75). The same study found that nausea and vomiting were more common with PCA fentanyl. Patients given IM meperidine were as sedated as those given sublingual buprenorphine after abdominal surgery (30). Intramuscular meperidine also resulted in higher rates of nausea, vomiting, respiratory depression, and hypotension compared to IV or IM nalbuphine after abdominal or orthopedic surgery $(32,67)$.

One study found that IV pentazocine resulted in increased nausea versus IV meperidine after abdominal surgery (28). When comparing IM meperidine to IM meptazinol after abdominal and orthopedic surgery, similar incidences of sedation, nausea, vomiting, confusion, pallor, and sweating were observed (29). Likewise, similar incidences of nausea and vomiting were also noted when compared to sublingual buprenorphine after laparotomy (60).

Intramuscular meperidine was shown to have similar rates of nausea, vomiting, dizziness, and headache as IM tilidine after tonsillectomy (76). There were no significant differences in sedation, nausea, and dizziness between transnasal butorphanol and IM meperidine after anal surgery (81). The use of PCA oxymorphone following Cesarean delivery caused more nausea and vomiting compared to PCA meperidine, with equal levels of sedation (54). Intramuscular metkephamid resulted in similar rates of nausea, vomiting, and headache compared to IM meperidine when used for pain relief after episiotomy (63).

\subsubsection{Meperidine versus Nonsteroidal Anti- inflammatory drugs}

When comparing IM meperidine with IM ketorolac, meperidine was associated with significantly higher rates of sedation after orthopedic and dental surgery (65), and nausea after abdominal surgery (33). Studies comparing meperidine to diclofenac after Cesarean section and laminectomy reported a similar incidence 
of side effects including nausea $(50-52,80)$, headache (80), epigastric pain (80), vomiting (49-52), pruritus (50), and postoperative ileus (49). Meperidine was associated with significantly higher levels of sedation when compared to diclofenac (50-52). One study compared IM meperidine to rectally-administered indomethacin after tonsillectomy and found no significant difference in the incidence of nausea, vomiting, dizziness, and headache (76).

\subsubsection{Meperidine versus Other Classes of Analgesics}

According to 2 studies, there was no difference in the incidence of adverse events when comparing parenteral administration of dipyrone with parenteral meperidine after laparotomy and gynecological surgery $(31,61)$, except for a significantly lower level of sedation in the IM dipyrone group (61). A study comparing IM meperidine vs IM ketamine following thoracic surgery reported significantly increased respiratory depression and heart rates in the meperidine group, but the mean arterial pressures remained unchanged (73). Other adverse reactions were evenly distributed over both groups (73).

Intravenous physostigmine salicylate resulted in higher levels of nausea when compared to IV meperidine, but physostigmine was associated with significantly lower levels of sedation than meperidine (34). When comparing IM meperidine with epidural bupivacaine plus fentanyl, meperidine resulted in significantly increased levels of sedation (37). Two studies that compared IM meperidine with epidural bupivacaine or epidural bupivacaine plus fentanyl showed no differences in the neonatal Apgar scores at 1 and 5 minutes $(36,38)$. Higher rates of one-time Apgar scores of less than 7 with meperidine were found in one study that compared it to submucous paracervical blockade with bupivacaine (39). Neonatal respiratory depression occurred more commonly with IM meperidine compared to epidural bupivacaine with fentanyl (37).

\subsection{Patient Satisfaction}

Patient satisfaction rates with epidural bupivacaine alone (35), epidural bupivacaine plus fentanyl $(24,37,38)$, IM ketorolac $(65,79)$, transnasal butorphanol (81), and oral diclofenac (52) were higher compared to meperidine. Patient satisfaction scores in those who received meperidine did not differ from those who received suppository diclofenac (51), PCA fentanyl (27), PCA oxymorphone (54), IM tramadol (37), IV nalbuphine
(32), oral methadone (48), IM meptazinol (29), or IM pentazocine (29). Moreover, patients who received IM meperidine were reportedly as satisfied as those who received IM/sublingual buprenorphine (30). Finally, 5 studies reported no difference in patient satisfaction when comparing morphine with meperidine $(27,53,54)$.

\section{Discussion}

\subsection{Summary of Evidence}

Meperidine is a type of phenylpiperidine used for the treatment of moderate to severe pain (82). This narrative review aimed to consolidate evidence derived from clinical studies published until the end of 2018 to review the analgesic efficacy and safety of using meperidine in managing postoperative and labor pain.

Based on the studies in this review, meperidine generally had similar or inferior analgesic efficacy compared to other analgesics in treating acute postoperative or labor pain. Only 5 of the 62 studies included in the review showed meperidine to be more efficacious than other analgesics. According to the study by Kølliker et al in 1972 (28), IV meperidine was found to be more efficacious than IV pentazocine for upper abdominal surgery, but the authors attributed this to the use of a lower dose of pentazocine $(15 \mathrm{mg})$, rather than the 25 - $\mathrm{mg}$ dose which is equipotent to the $50-\mathrm{mg}$ meperidine dose used in the study. Furthermore, it was unclear from the article how the investigators assessed pain in that study (28). Another study in 1986 showed that IV meperidine was associated with better analgesic efficacy than IV physostigmine following major abdominal surgery (34). Intravenous physostigmine's analgesic effect lasted only 15 to 30 minutes after injection, which is attributable to the drug's rapid distribution and elimination. They suggested the use of epidural or intrathecal physostigmine (34). The third study showed that the use of IM meperidine following laparoscopic ovarian cystectomy significantly lowered pain scores compared to PCA morphine, but the PCA morphine group had significantly higher patient satisfaction scores (58). In the fourth study, PCA meperidine had better analgesic efficacy than PCA tramadol following open-heart surgery (75). In the fifth study, IM meperidine was associated with greater pain relief than IM tramadol at 30 and 60 minutes after drug administration, but there were no differences after that (46). Careful interpretation of the efficacy data should be made considering the small sample sizes, different dosages, and various routes of administration across studies. 
Meperidine was generally associated with higher levels of sedation and respiratory depression compared to other analgesic drugs. It was associated with a similar or higher incidence of nausea and vomiting compared to other analgesics. Only 3 studies showed that meperidine had a better safety or side-effect profile compared to other analgesics. PCA meperidine caused fewer incidences of nausea and vomiting than PCA morphine or fentanyl after open-heart surgery (75), and PCA oxymorphone after Cesarean section (54).

The clinical use of meperidine has been restricted since the 1990s (7). In 2014, the US Joint Commission's Pain Management Standards recommended restricting the use of meperidine to short-term treatment of acute pain (83). According to a 2004 safety bulletin by the Institute for Safe Medication Practices Canada, oral meperidine was not recommended, and if prescribed, the duration of parenteral meperidine should be limited to 48 hours (84). Furthermore, it was recommended that meperidine be avoided in elderly patients (84). The Australian and New Zealand College of Anaesthetists and Faculty of Pain Medicine discouraged the use of meperidine in favor of other opioids for acute postoperative pain (14). The US Food and Drug Administration has also warned that meperidine may result in opioid addiction, abuse, respiratory depression, neonatal opioid withdrawal syndrome, and interact with cytochrome P450 3A4 and monoamine oxidase (MAO) inhibitors (85).

Although the first reports on meperidine-dependency date back as far as $1940(7,86)$, the reviewed studies did not report any cases of meperidine dependency or abuse. In addition, other concerns regarding meperidine included the issue of euphoria, serotonin syndrome due to drug interactions, and central nervous system toxicity (7). However, the clinical studies included in this review did not report the occurrence of these serious adverse events. These serious adverse events are generally rare. Many of the included studies had relatively small sample sizes and were probably not powered to detect these rare adverse events. Furthermore, patients with comorbid conditions that would have made them vulnerable to developing these side effects may not have met the inclusion/exclusion crite- ria for the randomized controlled trials. Therefore, the results of this systematic review do not really answer questions about the association between meperidine and serious adverse events for acute postoperative pain management in clinical practice.

\subsection{Limitations}

There were some limitations to this review. The majority of the clinical studies included younger patients, who were likely to have a lower risk of meperidine toxicity compared to older patients. Many of the included clinical studies were old, with some published in the 1970-80s. The older studies often reported a limited observation period (e.g., 24 hours). The sample sizes of the randomized studies were often less than 100 patients, and therefore probably underpowered to detect differences in the incidence of less common adverse effects. One notable limitation was that clinical trials comparing different routes of drug administration were included. For example, studies comparing IV meperidine against epidural bupivacaine were included. These studies were not excluded in order to provide a comprehensive review; however, such comparisons may lead to misleading conclusions about the analgesic efficacy of meperidine versus other analgesic drugs. In addition to the above, this narrative review was restricted to full text papers that could be retrieved.

\section{Conclusions}

In conclusion, this review article summarized the risk-benefit of meperidine for acute postoperative and labor pain control. The results suggest that other analgesic drugs with superior or equivalent analgesic efficacy and possibly fewer side effects are available. In light of these improved therapeutic options and with the data derived from this narrative review, meperidine should no longer be used as a primary analgesic intervention.

\section{Acknowledgments}

The study was supported by the Department of Anaesthesiology in the university where the study was conducted. There are no other external funding or conflicts of interests to be declared. 


\section{References}

1. Rodgers A, Walker N, Schug S, et al. Reduction of postoperative mortality and morbidity with epidural or spinal anaesthesia: Results from overview of randomised trials. BMJ 2000; 321:1493.

2. Beattie WS, Badner NH, Choi PT. Metaanalysis demonstrates statistically significant reduction in postoperative myocardial infarction with the use of thoracic epidural analgesia. Anesth Analg 2003; 97:919-920.

3. Kehlet $\mathrm{H}$, Jensen TS, Woolf $\mathrm{CJ}$. Persistent postsurgical pain: Risk factors and prevention. Lancet 2006; 367:1618-1625.

4. Andrews HL. Cortical effects of Demerol. J Pharmacol Exp Ther 1942; 76:89-94.

5. Garimella V, Cellini C. Postoperative pain control. Clin Colon Rectal Surg 2013; 26:191-196.

6. Ramsay MA. Acute postoperative pain management. Proc (Bayl Univ Med Cent) 2000; 13:244-247.

7. Buck ML. Is meperidine the drug that just won't die? J Pediatr Pharmacol Ther 2011; 16:167-169.

8. Demerol (meperidine hydrochloride, USP). Bridgewater, NJ: sanofiaventis U.S. LLC; 2010. www. accessdata.fda.gov/drugsatfda_docs/ label/2011/005010s05olbl.pdf. Date Accessed 11/20/2018.

9. Pethidine hydrochloride. Sydney, NSW, Australia: AstraZeneca Pty Ltd; 2018 \{AU: check date. Your source says 2018\}. www.guildlink.com.au/gc/ws/astra/ pi.cfm?product=apppethiıog1l. Date Accessed 11/20/2018.

10. Latta KS, Ginsberg B, Barkin RL. Meperidine: A critical review. Am J Ther 2002; 9:53-68.

11. Batterman RC. Clinical effectiveness and safety of a new synthetic analgesic drug, Demerol. JAMA Intern Med 1943; 71:345-356.

12. Olive JM, Masana L, Gonzalez J. Meperidine and reversible parkinsonism. Mov Disord 1994; 9:115-116.

13. Dai YH, Ou KL, Chu PW. Cerebellar and oculomotor dysfunction induced by rapid infusion of pethidine. BMJ Case Rep 2014; 2014:bcr2014203868.

14. Schug SA, Palmer GM, Scott DA, Halliwell R, Trinca J; APM:SE Working Group of the Australian and New Zealand College of Anaesthetists and Faculty of Pain Medicine. Acute Pain Management: Scientific Evidence. 4th ed. $^{\text {th }}$
Melbourne, Australia: Australian and New Zealand College of Anaesthetists. http://fpm.anzca.edu.au/documents/ apmse4_2015_final. Date Published 2015. Date Accessed 11/20/2018.

15. National Pain Center. The 2017 Canadian guideline for opioids for chronic noncancer pain. http://nationalpaincentre. mcmaster.ca/documents/Opioid\%20 GL\%2ofor\%2oCMAJ_olmay2017.pdf. Date Published 2017. Date Accessed 05/22/2019.

16. Faculty of Pain Medicine. Long term prescribing. The Royal College of Anaesthetists. www.rcoa.ac.uk/facultyof-pain-medicine/opioids-aware/ structured-approach-to-prescribing/ long-term-prescribing\#Documentation. \{AU: web link is not good; update\} Date Published 2019. Date Accessed 05/22/2019.

17. Kornitzer BS, Manace LC, Fischberg DJ, Leipzig RM. Prevalence of meperidine use in older surgical patients. Arch Surg 2006; 141:76-81.

18. Pan HH, Li CY, Lin TC, Wang JO, Ho ST, Wang KY. Trends and characteristics of pethidine use in Taiwan: A six-yearlong survey. Clinics (Sao Paulo) 2012; 67:749-755.

19. Leong WL, Sng BL, Sia AT. A comparison between remifentanil and meperidine for labor analgesia: A systematic review. Anesth Analg 2011; 113:818-825.

20. Salman N, Durukan AB, Gurbuz HA, et al. Comparison of effects of epidural bupivacaine and intravenous meperidine analgesia on patient recovery following elective abdominal aortic surgery. Med Sci Monit 2013; 19:347-352.

21. Raddi P, Nagalingaswamy VP, Khatib F, Wang Y, Chandra SBC. Comparison of interpleural bupivacaine and intravenous pethidine for postoperative pain relief following open cholecystectomy. Arch Med Sci 2009; 5:57-62.

22. Ebrahimifard F, Nooraei N. Postoperative pain after laparoscopic cholecystectomy: A randomized clinical trial comparing intraperitoneal bupivacaine versus intravenous pethidine. Surg Laparosc Endosc Percutan Tech 2013; 23:88-92.

23. Brownridge P, Frewin DB. A comparative study of techniques of postoperative analgesia following caesarean section and lower abdominal surgery. Anaesth Intensive Care 1985; 13:123-130.

24. Rimaitis K, Marchertiene I, Pavalkis D.
Comparison of two different methods of analgesia. Postoperative course after colorectal cancer surgery. Medicina (Kaunas) 2003; 39:129-137.

25. Kyzer S, Ramadan E, Gersch M, Chaimoff C. Patient-controlled analgesia following vertical gastroplasty: A comparison with intramuscular narcotics. Obes Surg 1995; 5:18-21.

26. Plummer JL, Owen H, Ilsley AH, Inglis S. Morphine patient-controlled analgesia is superior to meperidine patientcontrolled analgesia for postoperative pain. Anesth Analg 1997; 84:794-799.

27. Woodhouse A, Ward ME, Mather LE. Intra-subject variability in postoperative patient-controlled analgesia (PCA): Is the patient equally satisfied with morphine, pethidine and fentanyl? Pain 1999; 80:545-553.

28. Kolliker K. Pentazocine, pethidine and placebo for postoperative pain: An assessment by means of ventilatory measurements and subjective evaluation. Acta Anaesthesiol Scand 1972; 16:11-16.

29. Paymaster NJ. Analgesia after operation. A controlled comparison of meptazinol, pentazocine and pethidine. $\mathrm{Br}$ J Anaesth 1977; 49:1139-1146.

30. Carl P, Crawford ME, Madsen NB, Ravlo O, Bach V, Larsen Al. Pain relief after major abdominal surgery: A double-blind controlled comparison of sublingual buprenorphine, intramuscular buprenorphine, and intramuscular meperidine. Anesth Analg 1987; 66:142-146.

31. Patel CV, Koppikar MG, Patel MS, Parulkar GB. A double-blind comparison of parenteral dipyrone and pethidine in the treatment of post-operative pain. Curr Med Res Opin 1980; 6:624-629.

32. Hew E, Foster K, Gordon R, HewSang E. A comparison of nalbuphine and meperidine in treatment of postoperative pain. Can J Anaesth 1987; 34:462-465.

33. Folsland B, Skulberg A, Halvorsen $P$, Helgesen KG. Placebo-controlled comparison of single intramuscular doses of ketorolac tromethamine and pethidine for post-operative analgesia. J Int Med Res 1990; 18:305-314.

34. Petersson J, Gordh TE, Hartvig P, Wiklund L. A double-blind trial of the analgesic properties of physostigmine in postoperative patients. Acta Anaesthesiol 
Scand 1986; 30:283-288.

35. Harrison RF, Shore M, Woods $T$, Mathews G, Gardiner J, Unwin A. A comparative study of transcutaneous electrical nerve stimulation (TENS), entonox, pethidine + promazine and lumbar epidural for pain relief in labor. Acta Obstet Gynecol Scand 1987; 66:9-14.

36. Philipsen $\mathrm{T}$, Jensen NH. Epidural block or parenteral pethidine as analgesic in labour; a randomized study concerning progress in labour and instrumental deliveries. Eur J Obstet Gynecol Reprod Biol 1989; 30:27-33.

37. Jain S, Arya VK, Gopalan S, Jain V. Analgesic efficacy of intramuscular opioids versus epidural analgesia in labor. Int J Gynaecol Obstet 2003; 83:19-27.

38. Sharma SK, Sidawi JE, Ramin SM, Lucas MJ, Leveno KJ, Cunningham FG. Cesarean delivery: A randomized trial of epidural versus patient-controlled meperidine analgesia during labor. Anesthesiology 1997; 87:487-494.

39. Jensen F, Qvist I, Brocks V, Secher NJ, Westergaard LG. Submucous paracervical blockade compared with intramuscular meperidine as analgesia during labor: A double-blind study. Obstet Gynecol 1984; 64:724-727.

40. Ng TK, Cheng BC, Chan WS, Lam KK, Chan MT. A double-blind randomised comparison of intravenous patient-controlled remifentanil with intramuscular pethidine for labour analgesia. Anaesthesia 2011; 66:796-801.

41. Fleet J, Belan I, Jones MJ, Ullah S, Cyna AM. A comparison of fentanyl with pethidine for pain relief during childbirth: A randomised controlled trial. BJOG 2015; 122:983-992.

42. Elbohoty AE, Abd-Elrazek H, Abd-ElGawad M, Salama F, El-Shorbagy M, Abd-El-Maeboud $\mathrm{KH}$. Intravenous infusion of paracetamol versus intravenous pethidine as an intrapartum analgesic in the first stage of labor. Int ] Gynaecol Obstet 2012; 118:7-10.

43. Loughnan BA, Carli F, Romney M, Doré C), Gordon H. Randomized controlled comparison of epidural bupivacaine versus pethidine for analgesia in labour. $\mathrm{Br}$ J Anaesth 2000; 84:715-719.

44. Wee MY, TuckeyJP, Thomas PW, Burnard S. A comparison of intramuscular diamorphine and intramuscular pethidine for labour analgesia: A twocentre randomised blinded controlled trial. BJOG 2014; 121:447-456.
45. Douma MR, Verwey RA, Kam-Endtz $C E$, van der Linden PD, Stienstra R. Obstetric analgesia: A comparison of patient-controlled meperidine, remifentanil, and fentanyl in labour. $\mathrm{Br}$ ] Anaesth 2010; 104:209-215.

46. Keskin HL, Keskin EA, Avsar AF, Tabuk M, Caglar GS. Pethidine versus tramadol for pain relief during labor. Int J Gynaecol Obstet 2003; 82:11-16.

47. Tsui MH, Ngan Kee WD, Ng FF, Lau TK. A double blinded randomised placebocontrolled study of intramuscular pethidine for pain relief in the first stage of labour. BJOG 2004; 111:648-655.

48. Shahraki AD, Jabalameli $M$, Ghaedi $S$. Pain relief after cesarean section: Oral methadone vs. intramuscular pethidine. J Res Med Sci 2012; 17:143-147.

49. Rahmanpoor $H$, Hosseini $S N$ Mousavinasab S, Tadayon P, Karimi F. Comparison of diclofenac with pethidine on the pain after cesarean section. Int J Pharmacol 2007; 3:201-203.

50. Bozkurt N, Kurdoglu M, Kurdoglu Z, Kutlusoy F, Biberoglu K. Postoperative pain control after cesarean section: Can diclofenac sodium be used instead of meperidine? J Matern Fetal Neonatal Med 2009; 22:1144-1150.

51. Soroori ZZ, Sharami S, Heidarzadeh A, Leila S. The comparison between suppository diclofenac and pethidine in post-cesarean section pain relief: A randomized controlled clinical trial. J Res Med Sci 2006; 11:292-296.

52. Marzida M. A randomized controlled study comparing subcutaneous pethidine with oral diclofenac for pain relief after caesarean section. JUMMEC 2009; 12:63-69.

53. Cade L, Ashley J. Towards optimal analgesia after caesarean section: Comparison of epidural and intravenous patient-controlled opioid analgesia. Anaesth Intensive Care 1993; 21:696-699.

54. Sinatra RS, Lodge K, Sibert K, et al. A comparison of morphine, meperidine, and oxymorphone as utilized in patientcontrolled analgesia following cesarean delivery. Anesthesiology 1989; 70:585-590.

55. Stanley G, Appadu B, Mead M, Rowbotham DJ. Dose requirements, efficacy and side effects of morphine and pethidine delivered by patientcontrolled analgesia after gynaecological surgery. Br J Anaesth 1996; 76:484-486.

56. Ezri T, Lurie $S$, Stein A, Evron $S$, Geva D. Postoperative nausea and vomiting: Comparison of the effect of postoperative meperidine or morphine in gynecologic surgery patients. J Clin Anesth 2002; 14:262-266.

57. Unlugenc $H$, Vardar MA, Tetiker S. A comparative study of the analgesic effect of patient-controlled morphine, pethidine, and tramadol for postoperative pain management after abdominal hysterectomy. Anesth Analg 2008; 106:309-312, table of contents.

58. Bayar U, Basaran M, Atasoy N, Ayoglu $H$, Sade $H$, Altunkaya $H$. Comparison of satisfaction and pain relief between patients-controlled analgesia and interval analgesia after laparoscopic ovarian cystectomy. J Psychosom Obstet Gynaecol 2008; 29:139-145.

59. Shamim F, Hoda MQ, Samad K, Sabir S. Comparison between tramadol and pethidine in patient controlled intravenous analgesia. J Pak Med Assoc 2006; 56:433-436.

6o. Moa G, Zetterstrom H. Sublingual buprenorphine as postoperative analgesic: A double-blind comparison with pethidine. Acta Anaesthesiol Scand 1990; 34:68-71.

61. Bloch B, Smythe E, Weeks R. Analgesics for pain relief after gynaecological surgery. A two-phase study. S Afr Med J 1985; 67:325-329.

62. Colbert ST, Moran K, O'Hanlon DM, Chambers F, Moriarty DC, Blunnie WP. An assessment of the value of intraperitoneal meperidine for analgesia postlaparoscopic tubal ligation. Anesth Analg 2000; 91:667-670.

63. Bloomfield SS, Barden TP, Mitchell J. Metkephamid and meperidine analgesia after episiotomy. Clin Pharmacol Ther 1983; 34:240-247.

64. Tarradell R, Pol O, Farre M, Barrera E, Puig MM. Respiratory and analgesic effects of meperidine and tramadol in patients undergoing orthopedic surgery. Methods Find Exp Clin Pharmacol 1996; 18:211-218.

65. DeAndrade JR, Maslanka M, Reines HD, et al. Ketorolac versus meperidine for pain relief after orthopaedic surgery. Clin Orthop Relat Res 1996; 325:301-312.

66. Lindgren $U$, Djupsjo $H$. Diclofenac for pain after hip surgery. Acta Orthop Scand 1985; 56:28-31.

67. Brock-Utne JG, Ritchie P, Downing JW. A comparison of nalbuphine and pethidine for postoperative pain relief after orthopaedic surgery. S Afr Med J 1985; 68:391-393. 
68. Arti $\mathrm{H}$, Arti $\mathrm{S}$. The effects of intraarticular opioids in pain relief after arthroscopic menisectomy: A randomized clinical trial study. Pak J Med Sci 2013; 29:625-628.

69. Arti $\mathrm{H}$, Mehdinasab SA. The comparison effects of intra-articular injection of different opioids on postoperative pain relieve after arthroscopic anterior cruciate ligament reconstruction: A randomized clinical trial study.] Res Med Sci 2011; 16:1176-1182.

70. Lyons B, Lohan D, Flynn CG, Joshi GP, O'Brien TM, McCarroll M. Intraarticular analgesia for arthroscopic meniscectomy. $\mathrm{Br} J$ Anaesth 1995; 75:552-555.

71. Soderlund A, Westman L, Ersmark $H$, Eriksson E, Valentin A, Ekblom A. Analgesia following arthroscopy - a comparison of intra-articular morphine, pethidine and fentanyl. Acta Anaesthesiol Scand 1997; 41:6-11.

72. Saryazdi H, Kashefi P, Heydari M, Kiani A. Analgesic effects of intra-articular fentanyl, pethidine and dexamethasone after knee arthroscopic surgery. J Res Med Sci 2006; 11:156-159.

73. Dich-Nielsen JO, Svendsen LB, Berthelsen P. Intramuscular lowdose ketamine versus pethidine for postoperative pain treatment after thoracic surgery. Acta Anaesthesiol Scand 1992; 36:583-587.

74. Ohqvist G, Hallin R, Gelinder S, Lang $H$, Samuelson S. A comparison between morphine, meperidine and ketobemidone in continuous intravenous infusion for postoperative relief. Acta Anaesthesiol Scand 1991; 35:44-48.

75. Oztekin DS, Oztekin I, Issever H, Goksel O, Cinar B, Canik S. Postoperative effects of opioid analgesics administered via continuous perfusion and patient controlled analgesia after open heart surgery. Yakugaku Zasshi 2006; 126:499-504.

76. Saarnivaara L, Metsa-Ketela T, Mannisto P, Vapaatalo H. Pain relief and sputum prostaglandins in adults treated with pethidine, tilidine and indomethacin after tonsillectomy: A double-blind study. Acta Anaesthesiol Scand 1980; 24:79-85.

77. Sengezer $M$, Deveci $M$, Ozturk $S$, Bozkurt M, Kurt E, Suer AH. Two in one: Patient-controlled epidural analgesia (PCEA) to prevent erection and control pain in adult hypospadias-surgery patients. Br] Plast Surg 2002; 55:494-497.

78. Mojtahedzadeh M, Hashemian F, Najaf $A$, et al. Comparison of the analgesic profile and side effects of tramadol vs pethidine, following urologoical surgery. Daru 2004; 12:111-114.

79. Fricke JR Jr, Angelocci D, Fox K, McHugh $D$, Bynum L, Yee JP. Comparison of the efficacy and safety of ketorolac and meperidine in the relief of dental pain. J Clin Pharmacol 1992; 32:376-384.

80. Emamhadi MR, Hatamian HR. Comparing the effect of intramuscular injection of pethidine and diclofenac suppository in relief of pain following laminectomy surgery. Acta Med Iran 2008; 46:287-290.
81. Mai CM, Wan LT, Chou YC, et al. Efficacy and safety of transnasal butorphanol for pain relief after anal surgery. World J Gastroenterol 2009; 15:4829-4832.

82. Yasaei R, Saadabadi A. Meperidine. Meperidine. StatPearls Publishing LLC. www.ncbi.nlm.nih.gov/books/ NBK470362/. Date Updated 10/09/2019. Date Accessed 05/27/2019.

83. Joint Commission on Accreditation of Healthcare Organizations. Improving the quality of pain management through measurement and action. www.npcnow.org/system/files/research/ download/Improving-the-Qualityof-Pain-Management-ThroughMeasurement-and-Action.pdf. Date Published 03/2003. Date Accessed Date Accessed 05/27/2019.

84. Institute for Safe Medication Practices Canada (ISMP Canada). Meperidine (Demerol): Issues in medication safety. www.ismp-canada.org/download/ safetyBulletins/ISMPCSB2004-08.pdf. Date Published 08/2004. Date Accessed $11 / 20 / 2018$.

85. US National Library of Medicine. DEMEROL - meperidine hydrochloride injection, solution. DailyMed Web site. https://dailymed.nlm.nih.gov/dailymed/ drugInfo.cfm?setid=b31d1308-28c343f4-eoa6-2f3ed76b8975. Date Published 2018. Date Accessed 11/20/2018.

86. Rasor RW, Crecraft HJ. Addiction to meperidine (demerol) hydrochloride. J Am Med Assoc 1955; 157:654-657. 
